\title{
Clinical Insights Into Novel Immune Checkpoint Inhibitors
}

\author{
Jii Bum Lee ${ }^{1,2}$, Sang-Jun $\mathrm{Ha}^{3 *}$ and Hye Ryun $\mathrm{Kim}^{2 *}$ \\ ${ }^{1}$ Division of Hemato-oncology, Wonju Severance Christian Hospital, Yonsei University Wonju College of Medicine, Wonju, South \\ Korea, ${ }^{2}$ Division of Medical Oncology, Department of Internal Medicine, Yonsei Cancer Center, Yonsei University College of \\ Medicine, Seoul, South Korea, ${ }^{3}$ Department of Biochemistry, College of Life Science \& Biotechnology, Yonsei University, Seoul, \\ South Korea
}

The success of immune checkpoint inhibitors (ICls), notably anti-cytotoxic $\mathrm{T}$ lymphocyte associated antigen-4 (CTLA-4) as well as inhibitors of CTLA-4, programmed death 1 (PD1), and programmed death ligand-1 (PD-L1), has revolutionized treatment options for solid tumors. However, the lack of response to treatment, in terms of de novo or acquired resistance, and immune related adverse events (IRAE) remain as hurdles. One mechanisms to overcome the limitations of ICls is to target other immune checkpoints associated with tumor microenvironment. Immune checkpoints such as lymphocyte

OPEN ACCESS

Edited by:

Claudia Cerella,

Fondation de Recherche Cancer et

Sang, Luxembourg

Reviewed by:

Carmen Stecher,

Medical University of Vienna, Austria

Salman M Toor,

Hamad bin Khalifa University, Qatar

Reem Saleh,

Qatar Biomedical Research Institute,

Qatar

*Correspondence:

Hye Ryun Kim

nobelg@yuhs.ac

Sang-Jun $\mathrm{Ha}$

siha@yonsei.ac.kr

Specialty section: This article was submitted to Pharmacology of Anti-Cancer Drugs, a section of the journal

Frontiers in Pharmacology

Received: 16 March 2021

Accepted: 22 April 2021

Published: 06 May 2021

Citation:

Lee JB, Ha S-J and Kim HR (2021) Clinical Insights Into Novel Immune

Checkpoint Inhibitors.

Front. Pharmacol. 12:681320. doi: $10.3389 /$ fphar.2021.681320 activation gene-3 (LAG-3), T cell immunoglobulin and ITIM domain (TIGIT), T cell immunoglobulin and mucin-domain containing-3 (TIM-3), V-domain immunoglobulin suppressor of $\mathrm{T}$ cell activation (VISTA), B7 homolog 3 protein (B7-H3), inducible $T$ cell costimulatory (ICOS), and B and T lymphocyte attenuator (BTLA) are feasible and promising options for treating solid tumors, and clinical trials are currently under active investigation. This review aims to summarize the clinical aspects of the immune checkpoints and introduce novel agents targeting these checkpoints.

Keywords: immune checkpoint, LAG-3, TIGIT, TIM-3, B7-H3, VISTA, ICOS, BTLA

\section{BACKGROUND}

Cancer cells have characteristics that allow diversification and sustenance of their neoplastic state (Hanahan and Weinberg, 2011). One of the hallmarks of cancer is immune evasion; cancer cells hamper immune activation by limiting $\mathrm{T}$ cell activation and expressing immune checkpoint proteins on T cells (Vinay et al., 2015). Blocking cytotoxic T lymphocyte associated antigen-4 (CTLA-4) and the interaction between programmed death 1 (PD-1) and programmed death ligand-1 (PD-L1) elicit activation of the host immune system through T cell responses (Pardoll, 2012). These findings have led to the development of immune checkpoint inhibitors (ICIs) to control one of the key mechanisms utilized by cancer cells (Pardoll, 2012). In 2011, ipilimumab, the first anti-CTLA-4 monoclonal antibody (mAb), was approved for treating metastatic melanoma (Cameron et al., 2011). Thereafter, anti-PD-1 mAbs such as pembrolizumab, nivolumab, cemiplimab and as well as anti-PD-L1 mAbs such as atezolizumab, avelumab, durvalumab, have been used to treat patients with cancer, especially

\footnotetext{
Abbreviations: adhesion molecule 1; BTLA, B and T-lymphocyte attenuator; HVEM, herpes-virus entry mediator; ICOS, Inducible T cell costimulator; ICOSL, Inducible T cell costimulatory ligand; LAG-3, lymphocyte-associated gene 3; mAb, monoclonal antibody; PtdSer, phosphatidyl serine; TIGIT, T cell immunoglobulin and ITIM domain; TIM-3, T-cell immunoglobulin and mucin domain-3; VISTA, V-domain immunoglobulin suppressor of T cell activation; VSIG-3, V-Set and Immunoglobulin domain containing 3.
} 
in locally advanced and metastatic settings (Qin et al., 2019; Vaddepally et al., 2020). Besides PD-L1 expression, several emerging biomarkers have gained wide attention (Darvin et al., 2018). Pembrolizumab was approved in solid tumors harboring microsatellite instability-high (MSI-H) or mismatch repair deficient (dMMR), and high tumor mutation burden (TMB-H) defined as $\geq 10$ mutations/megabase based on FoundationOneCDx assay (Foundation Medicine, Inc.) (Marcus et al., 2019; Marabelle et al., 2020).

Despite the feasibility and anti-tumor activity of ICIs, there remain several hurdles in immunotherapy for cancer. Only a subset of patients respond to treatment, and the majority of patients who have durable responses eventually experience disease progression (Trebeschi et al., 2019). Furthermore, patients experience IRAE, some of which are highly toxic (Boutros et al., 2016; Wang et al., 2018). To overcome these impediments, treatment strategies such as combination with chemotherapy, targeted agents, or radiotherapy have been implemented (Gandhi et al., 2018; Wang et al., 2018; Rini et al., 2019). Notably, treatment with a combination of different ICIs has resulted in increased clinical responses, as observed with the combination of nivolumab and ipilimumab in melanoma, non-small cell lung cancer (NSCLC), and renal cell carcinoma (RCC) (Rizvi et al., 2016; Hellmann et al., 2018; Motzer et al., 2018).

Promising results from the combination of anti-CTLA-4 and PD-L1 mAbs have resulted in the launch of several other ICI combinations with non-overlapping mechanisms of action that may increase efficacy and minimize toxicity (Barbari et al., 2020). Currently, approximately $2 / 3$ of all oncology trials are dedicated to $\mathrm{T}$ cell-targeting immunomodulators, and there are more than 3,000 ongoing clinical trials (Xin Yu et al., 2019).

Resistance to immunotherapy is associated with loss of immunogenic neoantigens, increase of immunosuppressive cells, and upregulation of alternate immune checkpoint receptors (Sharma et al., 2017). This review provides an overview of the mechanisms and ongoing clinical trials specifically on novel emerging immune checkpoints, including lymphocyte activation gene-3 (LAG-3), T cell immunoglobulin and ITIM domain (TIGIT), T cell immunoglobulin and mucindomain containing-3 (TIM-3), V-domain immunoglobulin suppressor of $\mathrm{T}$ cell activation (VISTA), B7 homolog 3 protein (B7-H3), inducible $\mathrm{T}$ cell costimulatory (ICOS), and B and T lymphocyte attenuator (BTLA) (Chapoval et al., 2001; Monney et al., 2002; Yu et al., 2009; Paulos and June, 2010; Wang et al., 2011; Andrews et al., 2017; Marinelli et al., 2018).

\section{LAG-3}

LAG-3 is a protein comprising four parts-the hydrophobic, extracellular, transmembrane, and cytoplasmic domains. LAG-3 shares structural similarity with CD4 in having four extracellular regions (Triebel et al., 1990; Huard et al., 1997). It is expressed mainly on activated $\mathrm{CD}^{+}$and $\mathrm{CD}^{+} \mathrm{T}$ cells, regulatory T cells (Tregs), and natural killer (NK) cells, as well as on B cells and plasmacytoid dendritic cells (DCs) (Table 1)
(Huard et al., 1995; Andreae et al., 2002; Huang et al., 2004; Kisielow et al., 2005). LAG-3 binds its canonical ligand, major histocompatibility complex class II (MHC-II), as well as other ligands, including galectin-3, LSECtin, a-synuclein, and fibrinogen-like protein 1 (FGL1), thereby inducing exhaustion of immune cells and decreased cytokine secretion (Baixeras et al., 1992; Huard et al., 1994; Kouo et al., 2015; Anderson et al., 2016; Baumeister et al., 2016; Mao et al., 2016; Wang et al., 2019).

LAG-3 was found to be simultaneously co-expressed with other targets, such as PD-L1, TIGIT, and TIM-3, in preclinical settings (Woo et al., 2012; Baumeister et al., 2016). Blocking LAG3 alone did not restore $\mathrm{T}$ cell exhaustion; however, the combination of LAG-3/PD-1 blockade resulted in reduced tumor volume (Woo et al., 2012). These findings were consistent across in vivo studies using murine models of other tumors, including melanoma, ovarian cancer, and lymphoma (Goding et al., 2013; Huang et al., 2015).

In humans, LAG-3 is expressed on $\mathrm{CD}^{+}$tumor-infiltrating lymphocytes (TILs) and peripheral Tregs (Camisaschi et al., 2010; Matsuzaki et al., 2010; Li et al., 2013; Llosa et al., 2015; Taube et al., 2015). CD8 ${ }^{+}$TILs isolated from tumors such as hepatocellular carcinoma (HCC), melanoma, ovarian cancer, and microsatellite instability high (MSI) colorectal cancer (CRC), have high levels of both PD-1 and LAG-3 (Matsuzaki et al., 2010; Li et al., 2013; Llosa et al., 2015; Taube et al., 2015). Peripheral Tregs have been observed in melanoma and CRC (Camisaschi et al., 2010). In patients with hormone receptor-positive breast cancer, treated with immunotherapy, soluble LAG-3 (sLAG-3) detected in the serum was correlated with better prognosis in terms of disease-free survival (DFS) and overall survival (OS) (Triebel et al., 2006). However, the mechanism of sLAG-3 has yet to be identified ( $\mathrm{Li}$ et al., 2007).

\section{Clinical Trials on LAG-3}

Co-expression of LAG-3 with immune checkpoints, such as PD1 , and robust clinical data on the efficacy of LAG-3 and PD-1 dual blockade have prompted trials focusing on this combination as well as other immune checkpoint inhibitors. Currently, there are 17 agents targeting LAG-3 (Table 2), with multiple combinations of treatments across various tumors (Table 3). Eight of these agents have interim or final clinical results, and nine of the investigational agents are ongoing clinical trials.

A phase 1 study of eftilagimod alpha (IMP321), an antigenpresenting cell (APC) activator for LAG-3, in combination with pembrolizumab was conducted in 24 patients with metastatic melanoma (NCT02676869) (Atkinson et al., 2020). The primary endpoints were the recommended phase 2 dose (RP2D), safety, and tolerability of the combined agents. The study included cohort A of dose escalation and cohort B of extension, and the patients received subcutaneous pembrolizumab and eftilagimod alpha bi-weekly at doses of 1,6 , or $30 \mathrm{mg}$ for up to 6 and 12 months for Cohorts $\mathrm{A}$ and $\mathrm{B}$, respectively. There was no dose-limiting toxicity (DLT) and the treatment was well tolerated, with the injection site as the most common adverse event (AE). The response to treatment was encouraging, with an 
TABLE 1 | Overview of novel immune checkpoints.

\begin{tabular}{|c|c|c|c|c|c|c|c|}
\hline $\begin{array}{l}\text { Immune } \\
\text { checkpoints }\end{array}$ & LAG-3 & TIGIT & TIM-3 & B7-H3 & VISTA & Icos & BTLA \\
\hline Other names & CD223 & $\begin{array}{l}\text { Vstm3, Vsig9, } \\
\text { WUCAM }\end{array}$ & HAVCR2 & CD276 & $\begin{array}{l}\text { Dies1, DD1a, } \\
\text { Gi24, B7-H5, } \\
\text { PD-1H }\end{array}$ & CD278 & CD272 \\
\hline Function & Co-inhibition & Co-inhibition & Co-inhibition & $\begin{array}{l}\text { Co-inhibition or co- } \\
\text { stimulation }\end{array}$ & Co-inhibition & $\begin{array}{l}\text { Co-inhibition } \\
\text { or co- } \\
\text { stimulation }\end{array}$ & $\begin{array}{l}\text { Co-inhibition or } \\
\text { co-stimulation }\end{array}$ \\
\hline $\begin{array}{l}\text { Cells that express } \\
\text { the immune } \\
\text { checkpoints }\end{array}$ & $\begin{array}{l}\text { NK cells, DC, activated } \\
\text { T cells, Tregs, B cells, }\end{array}$ & $\begin{array}{l}\text { NK cells, } \\
\text { T cells }\end{array}$ & $\begin{array}{l}\text { NK cells, DCs, } \\
\text { activated T cells, } \\
\text { Tregs, B cells, } \\
\text { monocytes, cancer } \\
\text { cells }\end{array}$ & $\begin{array}{l}\text { NK cells, DCs, activated } \\
\text { T cells, monocytes, } \\
\text { cancer cells }\end{array}$ & $\begin{array}{l}\text { T cells, } \\
\text { myeloid cells }\end{array}$ & $\begin{array}{l}\text { Activated } \\
\text { T cells }\end{array}$ & $\begin{array}{l}\text { Mature T cells, } \\
\text { Tregs, B cells, } \\
\text { macrophages }\end{array}$ \\
\hline $\begin{array}{l}\text { Ligands or } \\
\text { receptors }\end{array}$ & $\begin{array}{l}\text { MHC-II, galectin-3, } \\
\text { LSECtin, a-synuclein, } \\
\text { FGL1 }\end{array}$ & $\begin{array}{l}\text { CD155, } \\
\text { CD112 }\end{array}$ & $\begin{array}{l}\text { HMGB-1, galectin- } \\
\text { 9, ceacam-1, } \\
\text { PtdSer }\end{array}$ & Unknown & VSIG-3 & ICOSL & $\begin{array}{l}\text { HVEM, LIGHT, } \\
\text { lymphotoxin- } \alpha\end{array}$ \\
\hline $\begin{array}{l}\text { Immune } \\
\text { checkpoint } \\
\text { agents }\end{array}$ & $\begin{array}{l}\text { APC activator, anti-LAG3 } \\
\text { mAb, LAG3 and PD1 DART } \\
\text { protein, LAG3 fusion } \\
\text { protein, bispecific Ab to } \\
\text { both LAG3 and PD-L1 }\end{array}$ & $\begin{array}{l}\text { Anti- } \\
\text { TIGIT mAb }\end{array}$ & $\begin{array}{l}\text { Anti-TIM-3 mAb, } \\
\text { anti-PD-1/TIM3 } \\
\text { bispecific Ab }\end{array}$ & $\begin{array}{l}\text { Anti-B7-H3 mAb, B7- } \\
\text { H3-targeting ADC, } \\
\text { radiolabeled anti-B7-H3 } \\
\text { mAb, CAR T-cell therapy }\end{array}$ & $\begin{array}{l}\text { Anti-VISTA } \\
\text { mAb, small } \\
\text { molecule } \\
\text { VISTA }\end{array}$ & $\begin{array}{l}\text { Anti-ICOS } \\
\text { agonist, anti- } \\
\text { ICOS } \\
\text { antagonist }\end{array}$ & \\
\hline $\begin{array}{l}\text { No. of } \\
\text { investigational } \\
\text { agents }\end{array}$ & 17 & 10 & 8 & 11 & 3 & 4 & 4 \\
\hline \multicolumn{8}{|l|}{ Clinical trials } \\
\hline Phase 1 & $\begin{array}{l}\text { Completed (eftilagimod } \\
\text { alpha, BI 754111, Sym022, } \\
\text { INCAGN02385), ongoing }\end{array}$ & Ongoing & $\begin{array}{l}\text { Completed } \\
\text { (Sym023), ongoing }\end{array}$ & $\begin{array}{l}\text { Completed } \\
\text { (enoblituzumab), } \\
\text { ongoing }\end{array}$ & $\begin{array}{l}\text { Completed } \\
\text { (CA-170), } \\
\text { ongoing }\end{array}$ & Ongoing & $\begin{array}{l}\text { Completed } \\
\text { (JTX-2011), } \\
\text { ongoing }\end{array}$ \\
\hline Phase 2 & $\begin{array}{l}\text { Completed (eftilagimod } \\
\text { alpha, LAG525), ongoing }\end{array}$ & Ongoing & Ongoing & Ongoing & NA & NA & NA \\
\hline Phase 3 & Ongoing (MGD013) & $\begin{array}{l}\text { Ongoing } \\
\text { (tiragolumab) }\end{array}$ & $\begin{array}{l}\text { Ongoing } \\
\text { (sabatolimab) }\end{array}$ & Ongoing & NA & NA & NA \\
\hline $\begin{array}{l}\text { Combination } \\
\text { treatment }\end{array}$ & Yes & Yes & Yes & Yes & No & Yes & Yes \\
\hline $\begin{array}{l}\text { Other immune } \\
\text { checkpoint } \\
\text { inhibitors }\end{array}$ & Yes & Yes & Yes & Yes & & Yes & Yes \\
\hline Targeted agents & Yes & Yes & Yes & Yes & & Yes & Yes \\
\hline Chemotherapy & Yes & Yes & Yes & Yes & & Yes & No \\
\hline Radiotherapy & Yes & No & No & Yes & & No & No \\
\hline
\end{tabular}

Abbreviations: APC, antigen presenting cell; BTLA, B and T-lymphocyte attenuator; CAR-T, chimeric antigen receptor T cell; DART, dual-affinity re-targeting proteins; DCs, dendritic cells; Dies 1, differentiation of embryonic stem cells 1; HAVCR2, hepatitis A virus cellular receptor 2; HVEM, herpes-virus entry mediator; mAb, monoclonal antibody; ICOS, Inducible T cell costimulator; ICOSL, Inducible T cell costimulatory ligand; LAG-3, lymphocyte-associated gene 3; NK cells, natural killer cells; PD-1H, PD-1 homologue; PD-L1, programmed death-ligand 1; PtdSer, phosphatidyl serine; T regs, ceacam-1, carcinoembryonic antigen cell adhesion molecule 1; T regs, regulatory T cells; TIGIT, T cell immunoglobulin and ITIM domain; TIM-3, T-cell immunoglobulin and mucin domain-3; VISTA, V-domain immunoglobulin suppressor of T cell activation; VSIG-3, V-Set and Immunoglobulin domain containing 3; WUCAM, Washington University cell adhesion molecule.

overall response rate (ORR) of 33 and $50 \%$ for pembrolizumabrefractory cohort $\mathrm{A}$ and $\mathrm{PD}-1$ naive cohort $\mathrm{B}$ patients, respectively.

Similarly, the combination of eftilagimod alpha and pembrolizumab has been investigated in NSCLC and head and neck squamous cell carcinoma (HNSCC) (NCT03625323) (Peguero et al., 2019). The AIPAC study, a placebo-controlled randomized phase IIb study on eftilagimod alpha (or placebo) with paclitaxel as the first-line treatment in patients with metastatic breast cancer (MBC), is also under investigation (NCT02614833) (Dirix and Triebel, 2019). Preliminary results show that the agent could elicit durable immune responses. Clinical data, including progression-free survival (PFS), ORR, OS, and safety, are all awaiting results.
Relatlimab (BMS-986016), an IgG4 mAb targeting LAG-3, has been investigated in various settings and agents, notably with well-established immune checkpoint inhibitors such as nivolumab and ipilimumab and other novel agents such as indoleamine 2,3-dioxygenase-1 (IDO1) inhibitors, CCR2/5 dual antagonist, and anti-TIGIT. Notably, clinical trials are ongoing for phase II/III in previously untreated metastatic melanoma, in combination with or without nivolumab (NCT03470922), phase II of nivolumab and oxaliplatin-based chemotherapy with or without relatlimab in GC or gastroesophageal junction (GEJ) cancer (NCT03662659), and phase II of relatlimab with nivolumab in mismatch repair deficient (dMMR) cancers resistant to prior PD-1/PD-L1 inhibition (Lipson et al., 2018; Feeney et al., 2019; Bever et al., 
TABLE 2 | Emerging immune checkpoint inhibitors and their mechanisms.

\begin{tabular}{|c|c|c|c|}
\hline Target & Name of agent & Company & Mechanism \\
\hline \multicolumn{4}{|l|}{ LAG-3 } \\
\hline & Eftilagimod alpha (IMP321) & Immutep & APC activator \\
\hline & Relatlimab (BMS-986016) & Bristol-Myers Squibb & $\lg \mathrm{G} 4 \mathrm{mAb}$ \\
\hline & LAG525 & Norvatis & $\operatorname{lgG} 4 \mathrm{mAb}$ \\
\hline & Cemiplimab (REGN3767) & Regeneron & $\mathrm{mAb}$ \\
\hline & BI 754111 & Bohringer Ingelheim & $\mathrm{mAb}$ \\
\hline & Sym022 & Symphogen & Fc-inert mAb \\
\hline & MGD013 & MacroGenics & LAG-3 and PD1 DART protein \\
\hline & Mavezelimab (MK-4280) & Merck & $\operatorname{lgG} 4 \mathrm{mAb}$ \\
\hline & TSR-033 & Tesaro & $\lg \mathrm{G} 4 \mathrm{mAb}$ \\
\hline & INCAGN02385 & Incyte & Fc engineered lgG1k antibody \\
\hline & EOC202 & EddingPharm Oncology & LAG-3 fusion protein \\
\hline & 89Zr-DFO-REGN3767 & Memorial Sloan Kettering Cancer Center & Anti-LAG-3 antibody labeled with $89 Z$ r \\
\hline & $\mathrm{XmAb}{ }^{\circledR} 22,841$ & Xencor & Bispecific antibody to both LAG3 and CTLA-4 \\
\hline & LBL-007 & Nanjing Leads Biolabs Co & AlphaLAG-3 mAb \\
\hline & FS118 & F-star & Bispecific antibody to both LAG3 and PD-L1 \\
\hline & RO7247669 & Hoffmann-La Roche & Bispecific antibody to both LAG3 and PD-L1 \\
\hline & EMB-02 & Shanghai EpimAb Biotherapeutics & Bispecific antibody to both LAG3 and PD-L1 \\
\hline \multicolumn{4}{|r|}{ 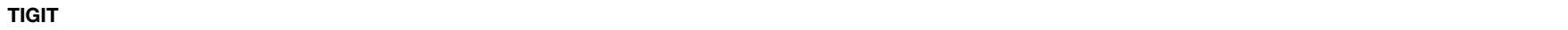 } \\
\hline & Tiragolumab (MTIG7192A/RG-6058) & Genentech & Anti-TIGIT mAb \\
\hline & Vibostolimab (MK-7684) & Merck & Anti-TIGIT mAb \\
\hline & Etigilimab (OMP-313M32) & OncoMed & Anti-TIGIT mAb \\
\hline & BMS-986207 & Bristol-Myers Squibb & Anti-TIGIT mAb \\
\hline & Domvanalimab (AB-154) & Arcus Biosciences & Anti-TIGIT mAb \\
\hline & ASP-8374 & Potenza & Anti-TIGIT mAb \\
\hline & IBI939 & Innovent Biologics & Anti-TIGIT mAb \\
\hline & BGB-A1217 & BeiGene & Anti-TIGIT mAb \\
\hline & COM902 & Compugen & Anti-TIGIT mAb \\
\hline & M6223 & EMD Serono & Anti-TIGIT mAb \\
\hline \multicolumn{4}{|l|}{ TIM-3 } \\
\hline & Sym023 & Symphogen & Anti-TIM-3 mAb \\
\hline & LY3321367 & Eli Lilly and Company & Anti-TIM-3 mAb \\
\hline & Cobolimab (TSR-022) & Tesaro & Anti-TIM-3 mAb \\
\hline & Sabatolimab (MBG453) & Novartis & Anti-TIM-3 mAb \\
\hline & INCAGN2390 & Incyte & Anti-TIM-3 mAb \\
\hline & BMS-986258 & Bristol-Myers Squibb & Anti-TIM-3 mAb \\
\hline & SHR-1702 & Jiangsu HengRui & Anti-TIM-3 mAb \\
\hline & $\mathrm{RO} 7121661$ & Roche & Anti-PD-1/TIM-33 bispecific Ab \\
\hline \multicolumn{4}{|l|}{ В7-H3 } \\
\hline & Enoblituzumab (MGA271) & MacroGenetics & Anti-B7-H3 mAb \\
\hline & DS-7300a & Daiichi Sankyo & B7-H3-targeting ADC \\
\hline & Orlotamab (MGD009) & MacroGenetics & $\mathrm{B} 7-\mathrm{H} 3$ and CD3 DART protein \\
\hline & 131I-Omburtamab & Y-mAbs Therapeutics & Radiolabeled anti-B7-H3 mAb \\
\hline & 124I-Omburtamab & Y-mAbs Therapeutics & Radiolabeled anti-B7-H3 mAb \\
\hline & 177Lu-DTPA-Omburtamab & Y-mAbs Therapeutics & Radiolabeled anti-B7-H3 mAb \\
\hline & 4SCAR-276 & Shenzhen Geno-Immune Medical Institute & CAR T-cell therapy \\
\hline & SCRI-CARB7H3 & Seattle Children's Hospital & CAR T-cell therapy \\
\hline & B7-H3 CAR-T & BoYuan RunSheng Pharma & CAR T-cell therapy \\
\hline & CAR.B7-H3 & UNC Lineberger Comprehensive Cancer Center & CAR T-cell therapy \\
\hline & Second-generation 4-1BB & Seattle Children's Hospital & CAR T-cell therapy \\
\hline \multicolumn{4}{|c|}{ 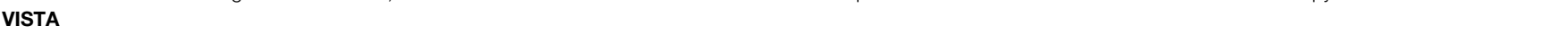 } \\
\hline & JNJ-61610588 & Johnson \& Johnson & Anti-VISTA mAb \\
\hline & $\mathrm{Cl}-8993$ & Curis & Anti-VISTA mAb \\
\hline & CA-170 & Curis & Small molecule targeting VISTA and PD-L1 \\
\hline \multicolumn{4}{|r|}{ 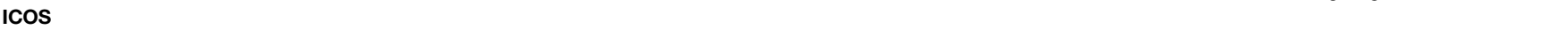 } \\
\hline & GSK3359609 & GlaxoSmithKline & Anti-ICOS agonist \\
\hline & JTX-2011 & Jounce Therapeutics & Anti-ICOS agonist \\
\hline & MEDI-570 & National Cancer Institute & Anti-ICOS antagonist \\
\hline & KY1044 & Kymab Limited & Anti-ICOS antagonist \\
\hline \multicolumn{4}{|l|}{ BTLA } \\
\hline & INBRX-106 & Inhibrx & Hexavalent $\mathrm{OX} 40$ agonist $\mathrm{Ab}$ \\
\hline & PF-04518600 & Pfizer & OX40 agonist \\
\hline & Cudarolimab (IBI101) & Innovent Biologics & Anti-OX40 mAb \\
\hline & TAB004 (JS004) & Shanghai Junshi Bioscience & Anti-BTLA mAb \\
\hline
\end{tabular}

Abbreviations: ADC, antibody drug conjugate; APC, antigen-presenting cell; BTLA, B and T-lymphocyte attenuator; CAR, chimeric antigen receptor; CTLA-4, cytotoxic T-lymphocyteassociated protein; DART, dual-affinity re-targeting proteins; ICOS, inducible T-cell costimulator; LAG3, lymphocyte-associated gene 3; mAb, monoclonal antibody; PD-L1, programmed death-ligand 1; TIGIT, T cell immunoglobulin and ITIM domain; TIM, T-cell immunoglobulin and mucin domain-3; VISTA, V-domain immunoglobulin suppressor of T cell activation. 
2020). Relatlimab is being tested in a wide range of tumor types and settings as front- or second-line treatment, in resectable status, and in stage II/III.

An open label, phase 2 study including 72 patients treated with LAG-525, which is an IgG4 mAb for LAG-3, and spartalizumab (PDR001), an anti-PD-1, for advanced solid tumors and hematologic malignancies showed promising activity, especially in neuroendocrine tumors, small cell lung cancer (SCLC), and diffuse large B-cell lymphoma (DLBCL), with a clinical benefit rate at 24 weeks (CBR24) of $0.86,0.27$, and 0.804 , respectively, meeting its primary endpoint (NCT03365791) (Uboha et al., 2019). In GEJ cancer, the CBR24 was 0.071, and enrollment was stopped for these subsets of patients. Other tumors such as triple-negative breast cancer (TNBC) (NCT03742349 and NCT03499899) and melanoma (NCT03484923) are ongoing trials in advanced and metastatic settings.

The preliminary results of a phase 1 study on cemiplimab (REGN3767), an mAb for LAG-3, as monotherapy $(n=27)$, and in combination with $\mathrm{PD}-1 \mathrm{mAb}(n=42)$ was conducted in advanced malignancies (NCT03005782) (Papadopoulos et al., 2019). No DLT was observed with in the monotherapy group, whereas the combination group, during treatment with R3767 $3 \mathrm{mg} / \mathrm{kg}$ every 3 weeks $(\mathrm{Q} 3 \mathrm{~W})+$ cemiplimab $3 \mathrm{mg} / \mathrm{kg}$ Q3W, experienced grade 4 elevated creatine phosphokinase levels in addition to grade 3 myasthenia gravis. Overall, both treatments were deemed tolerable; cemiplimab $20 \mathrm{mg} / \mathrm{kg}$ or $1600 \mathrm{mg}$ as a fixed dose of Q3W is ongoing further evaluation as monotherapy and as a combination.

Similarly, BI 754111, an mAb for LAG-3, was also tested with BI 754091 (anti-PD-1) in treatment-refractory solid tumors, in a dose escalation phase 1 study, followed by an expansion phase in microsatellite stable (MSS) CRC and anti-PD1/PD-L1 refractory tumors including NSCLC (NCT03156114) (Johnson et al., 2020). The primary endpoints for dose escalation and dose expansion phase were DLT and the maximum tolerated dose (MTD) and ORR, respectively. Biomarker analysis was performed in MSS CRC refractory to immunotherapy; the patients who responded to these agents with a partial response (PR) or stable disease (SD) had increased treatment-associated IFN- $\gamma$ gene signature scores (Bendell et al., 2020). Furthermore, patients with high PD-L1 gene expression in pre-treatment biopsy samples responded better to the treatment. Baseline immunohistochemistry of LAG-3 was not a predictive factor for this subset of patients.

Sym022 (anti-LAG-3) was evaluated as a single agent or in combination with sym021 (anti-PD-1) in phase 1 trials for solid tumors or lymphomas (NCT03311412, NCT03489369, and NCT03489343) (Lakhani et al., 2020). Interim analysis showed that 15 patients who were administered monotherapy and 20 patients under combination treatment, had one unconfirmed PR. Both treatment arms had tolerable safety profiles, with the combination treatment showing one grade 3-4 immune-related hypophysitis. Further assessments of the pharmacokinetic (PK) and pharmacodynamic (PD) markers and the anti-tumor activity of the monotherapy and combination are awaiting results.

MGD013 is a LAG-3 and PD-1 dual-affinity re-targeting (DART) protein; its safety, tolerability, DLT, MTD, PK/PD, and antitumor activity were analyzed in patients with unresectable and metastatic tumors in a phase 1 study (NCT03219268) (Luke et al., 2020). Fifty patients in the doseescalation phase and 157 patients in the dose-expansion phase, with 46 and $32 \%$ of patients with prior exposure to immunotherapy, respectively, were enrolled. No MTD was reached, and the most common treatment-related adverse events (TRAE), which were fatigue and nausea, were well tolerated. Despite exposure to previous immunotherapy, both cohorts included patients with objective responses. More mature clinical data are awaiting results, and biomarker analysis of LAG3 and PD-L1 is ongoing.

Other agents that are undergoing clinical trials are: 1) mavezelimab (MK-4280), an IgG4 mAb targeting LAG-3 (NCT03598608, NCT02720068, and NCT03516981); 2) TSR033, an IgG4 mAb targeting LAG-3 (NCT03250832); 3) INCAGN02385, a Fc engineered IgG1k antibody for LAG-3 (NCT03538028, NCT04370704, and NCT03311412); 4) EOC202, a LAG-3 fusion protein (NCT03600090); 5) 89ZrDFO-REGN3767, an anti-LAG-3 antibody labeled with $89 \mathrm{Zr}$ (NCT04566978); 6) $\mathrm{XmAb}^{\circledR} 22841$, a bispecific antibody to both LAG-3 and CTLA-4 (NCT03849469); 7) LBL-007, an alphaLAG-3 mAb (NCT04640545), and 8) bispecific antibody to both LAG-3 and PD-L1, which includes agents FS118 (NCT03440437), RO7247669 (NCT04140500), and EMB-02 (NCT04618393) treated as monotherapy or in combination for patients with treatment refractory solid and/or hematologic malignancies.

\section{TIGIT}

TIGIT, previously known as Vstm3, VSIG9, or Washington University cell adhesion molecule (WUCAM), is a protein comprising an extracellular IgV domain and an intracellular domain with a canonical ITIM and an immunoglobulin tyrosine tail (ITT) motif (Table 1) (Yu et al., 2009; Levin et al., 2011). TIGIT expression is tightly restricted to lymphocytes and is mainly observed in NK cells and T cell subsets, including effector and regulatory $\mathrm{CD}^{+} \mathrm{T}$ cells, follicular helper $\mathrm{CD} 4^{+} \mathrm{T}$ cells, and effector $\mathrm{CD}^{+} \mathrm{T}$ cells (Boles et al., 2009; Yu et al., 2009; Lozano et al., 2012; Stengel et al., 2012; Johnston et al., 2014; Joller et al., 2014). Three ligands bind to TIGIT: 1) poliovirus receptor (PVR), also known as CD155, Necl5, and Tage4; 2) CD112, also called poliovirus receptor ligand2/nectin2 (PVRL2/nectin 2); and 3) PVRL3. PVR has a high affinity for TIGIT, whereas CD112 and PVRL3 bind to a lesser extent (Yu et al., 2009).

TIGIT plays multiple roles in the inhibition of cancer immunity. TIGIT inhibits NK cell-mediated tumor killing, induces immunosuppressive DCs, suppresses CD8 T cell priming and differentiation, and prevents CD8 $\mathrm{T}$ cell-mediated killing (Buisson and Triebel, 2005; Li et al., 2014; Fuhrman et al., 2015; Kurtulus et al., 2015; Liu et al., 2015; Kourepini et al., 2016). The interaction of TIGIT with other constituents of the tumor microenvironments (TMEs), such as cancer-associated fibroblasts and angiogenesis, remains to be elucidated (Manieri et al., 2017). 
TABLE 3 | Clinical trials on novel immune checkpoint inhibitors.

\begin{tabular}{|c|c|c|c|c|c|}
\hline Target & Drug & $\begin{array}{l}\text { Clinical } \\
\text { trial no. }\end{array}$ & Phase & Settings & Tumor types \\
\hline \multirow[t]{33}{*}{ LAG-3 } & $\begin{array}{l}\text { Eftilagimod alpha } \\
\text { (IMP321) }\end{array}$ & NCT03252938 & 1 & Advanced/metastatic & Solid tumors \\
\hline & & NCT00351949 & 1 & Advanced/metastatic & $\mathrm{RCC}$ \\
\hline & & NCT00349934 & 1 & First line & Breast cancer \\
\hline & & NCT02614833 & 2 & Advanced/metastatic & Breast cancer \\
\hline & & NCT00324623 & 1 & Advanced/metastatic & Melanoma \\
\hline & & NCT00365937 & 1,2 & Adjuvant & Melanoma \\
\hline & & NCT01308294 & 1,2 & Stage II-IV & Melanoma \\
\hline & & NCT00732082 & 1 & Advanced/metastatic & Pancreatic cancer \\
\hline & & NCT02676869 & 1 & Stage III-IV & Melanoma \\
\hline & & NCT03625323 & 2 & Advanced/metastatic & NSCLC and HNSCC \\
\hline & Relatlimab (BMS-986016) & NCT02966548 & 1 & Advanced/metastatic & Solid tumors \\
\hline & & NCT01968109 & 1,2 & First, second line & Solid tumors \\
\hline & & NCT03623854 & 2 & Advanced/metastatic & Chordoma \\
\hline & & NCT03743766 & 2 & Advanced/metastatic & Melanoma \\
\hline & & NCT03470922 & 2,3 & Advanced/metastatic & Melanoma \\
\hline & & NCT03642067 & 2 & Advanced/metastatic & MSS CRC \\
\hline & & NCT04658147 & 1 & Resectable & $\mathrm{HCC}$ \\
\hline & & NCT02061761 & 1,2 & Advanced/metastatic & Hematologic malignancies \\
\hline & & NCT04567615 & 2 & Advanced/metastatic & $\mathrm{HCC}$ \\
\hline & & NCT03607890 & 2 & $\begin{array}{l}\text { Advanced, prior PD-(L)1 } \\
\text { inhibitor }\end{array}$ & MSI-H solid tumors \\
\hline & & NCT04326257 & 2 & $\begin{array}{l}\text { Advanced, prior PD-(L)1 } \\
\text { inhibitor }\end{array}$ & HNSCC \\
\hline & & NCT03493932 & 1 & Recurrent & Glioblastoma \\
\hline & & NCT02658981 & 1 & Recurrent & Glioblastoma \\
\hline & & NCT03610711 & 1,2 & Advanced/metastatic & GC, GEJ cancer \\
\hline & & NCT03044613 & 1 & Stage $\|/\| I \|$ & GC, GEJ cancer \\
\hline & & NCT03662659 & 2 & Advanced/metastatic & GC, GEJ cancer \\
\hline & & NCT03335540 & 1,2 & Advanced/metastatic & Solid tumors \\
\hline & & NCT04611126 & 1,2 & Advanced/metastatic & Ovarian cancer \\
\hline & & NCT02488759 & 1,2 & $\begin{array}{l}\text { Neoadjuvant and } \\
\text { metastatic }\end{array}$ & Virus-associated tumors \\
\hline & & NCT02519322 & 2 & Neoadjuvant and adjuvant & Melanoma \\
\hline & & NCT03459222 & 2 & Advanced/metastatic & Solid tumors \\
\hline & & NCT02996110 & 2 & Advanced/metastatic & $\mathrm{RCC}$ \\
\hline & & NCT02935634 & 2 & Advanced/metastatic & GC, GEJ cancer \\
\hline
\end{tabular}

$\begin{array}{lc} & \text { Treatment arms } \\ & \\ \text { Eftilagimod alpha } & \text { Activ } \\ \text { Eftilagimod alpha } & \text { recul } \\ \text { Eftilagimod alpha } & \text { C } \\ \text { Eftilagimod alpha } & \text { Active } \\ \text { Cyclophosphamide, fludarabine followed by melan-A VLP vaccine and } & \text { Co } \\ \text { eftilagimod alpha } & \\ \text { Eftilagimod alpha } \pm \text { HLA-A2 peptides } & \text { Te }\end{array}$

Eftilagimod alpha $\pm \mathrm{HLA}-\mathrm{A} 2$ peptides

Eftilagimod alpha+pembrolizumab

Terminated

Eftilagimod alpha+pembrolizumab

$\begin{array}{ll}\text { Relatlimab } \pm \text { nivolumab } & \text { Recruiting } \\ \text { Recruiting }\end{array}$

Relatlimab \pm nivolumab Recruiting

Relatlimab+nivolumab _recruiting

Relatlimab+nivolumab Recruiting

$\begin{array}{ll}\text { Relatlimab } \pm \text { nivolumab } & \text { Recruiting }\end{array}$

Relatlimab+nivolumab $\quad$ Recruiting

Relatlimab \pm nivolumab $\quad$ Not yet

Not yet

$\begin{array}{ll}\text { Relatlimab+nivolumab } & \text { Not yet } \\ & \text { recruiting }\end{array}$

Relatlimab+nivolumab

Recruiting

Relatlimab+nivolumab or ipilimumab

Recruiting

Relatlimab+nivolumab

Relatlimab \pm nivolumab

Active, not

Nivolumab, carboplatin, paclitaxel, radiation \pm relatlimab Recruiting

Relatlimab or nivolumab \pm investigator's choice of chemotherapy $\quad$ Active, not

Relatlimab+nivolumab or cabiralizumab or ipilimumab or IDO1 inhibitor or recruiting

radiation therapy

Relatlimab, nivolumab, cyclophosphamide, fludarabine phosphate, tumor Not yet

infiltrating lymphocytes infusion \pm ipilimumab recruiting

Nivolumab \pm relatlimab or ipilimumab or daratumumab Active, not

Nivolumab+relatlimab or ipilimumab

Recruiting

Recruiting

Nivolumab+ipilimumab or BMS-986205 (IDO1) or BMS-813160 (CCR2/5 Recruiting

dual antagonist)

Nivolumab \pm relatlimab or ipilimumab or rucaparib or BMS-986205; Recruiting

ipilimumab+ucaparib; nivolumab+ipilimumab+rucaparib 
TABLE 3 | (Continued) Clinical trials on novel immune checkpoint inhibitors.

\begin{tabular}{|c|c|c|c|c|c|c|c|}
\hline Target & Drug & $\begin{array}{l}\text { Clinical } \\
\text { trial no. }\end{array}$ & Phase & Settings & Tumor types & Treatment arms & Status \\
\hline & & NCT02750514 & 2 & Advanced/metastatic & NSCLC & Nivolumab \pm relatlimab or ipilimumab or BMS-986205 or dasatinib & $\begin{array}{l}\text { Active, not } \\
\text { recruiting }\end{array}$ \\
\hline & & NCT02060188 & 2 & Advanced/metastatic & $\mathrm{CRC}$ & Nivolumab \pm relatimab or daratumumab or ipilimumab \pm cobimetinib & $\begin{array}{l}\text { Active, not } \\
\text { recruiting }\end{array}$ \\
\hline & & NCT04150965 & 1,2 & Advanced/metastatic & Multiple myeloma & $\begin{array}{l}\text { Relatlimab } \pm \text { pomalidromide and dexamethasone; BMS-986207 (anti- } \\
\text { TIGIT) } \pm \text { pomalidromide and dexamethasone; elotuzumab }\end{array}$ & Recruiting \\
\hline \multirow{5}{*}{\multicolumn{2}{|c|}{ LAG525 }} & NCT02460224 & 1,2 & Advanced/metastatic & Solid tumors & LAG525 \pm spartalizumab (PDR001) & $\begin{array}{l}\text { Active, not } \\
\text { recruiting }\end{array}$ \\
\hline & & NCT03365791 & 2 & Advanced/metastatic & $\begin{array}{l}\text { Solid or hematologic } \\
\text { malignancy }\end{array}$ & LAG525+spartalizumab & Completed \\
\hline & & NCT03742349 & 1 & Advanced/metastatic & TNBC & $\begin{array}{l}\text { LAG525+spartalizumab+NIR178 or capmatinib or lacnotuzumab } \\
\text { (MCS110) or canakinumab }\end{array}$ & Recruiting \\
\hline & & NCT03499899 & 2 & Advanced/metastatic & TNBC & 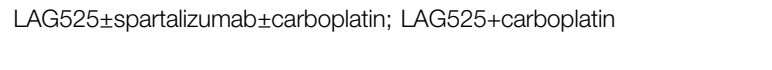 & $\begin{array}{l}\text { Active, not } \\
\text { recruiting }\end{array}$ \\
\hline & & NCT03484923 & 2 & Advanced/metastatic & Melanoma & Spartalizumab+lag525 or ribociclib or canakinumab or capmatinib & Recruiting \\
\hline & Cemiplimab (REGN3767) & NCT03005782 & 1 & Advanced/metastatic & Solid tumors or lymphomas & 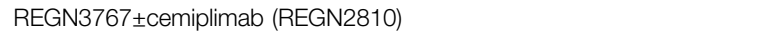 & Recruiting \\
\hline \multirow{5}{*}{\multicolumn{2}{|c|}{ Bl 754111}} & NCT03433898 & 1 & Advanced/metastatic & Solid tumors & BI $754111 \pm B \mid 754091$ (anti-PD-1) & Recruiting \\
\hline & & NCT03156114 & 1 & Advanced/metastatic & Solid tumors & Bl $754111+B \mid 754091$ & $\begin{array}{l}\text { Active, not } \\
\text { recruiting }\end{array}$ \\
\hline & & NCT03780725 & 1 & Advanced/metastatic & NSCLC and HNSCC & Bl $754111+B \mid 754091$ & Completed \\
\hline & & NCT03697304 & 2 & Advanced/metastatic & Solid tumors & $\begin{array}{l}\text { Bl } 754111 \text { or Bl } 836880 \text { (bispecific VEGF and Ang2 Ab)+BI } 754091 \text { (anti- } \\
\text { PD-1) }\end{array}$ & Recruiting \\
\hline & & NCT03964233 & 1 & Advanced/metastatic & Solid tumors & 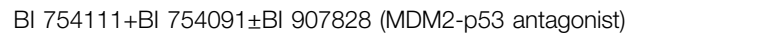 & Recruiting \\
\hline & Sym022 & NCT03489369 & 1 & Advanced/metastatic & Solid tumors or lymphomas & & Completed \\
\hline \multirow{2}{*}{\multicolumn{2}{|c|}{ MGD013 }} & NCT03219268 & 1 & Advanced/metastatic & $\begin{array}{l}\text { Solid or hematologic } \\
\text { malignancy }\end{array}$ & MGD013+margetuximab (anti-HER2 monoclonal antibody) & Recruiting \\
\hline & & NCT04082364 & 2,3 & Advanced/metastatic & GC, GEJ cancer & $\begin{array}{l}\text { margetuximab+INCMGA00012 (anti-PD-1); } \\
\text { margetuximab+chemotherapy } \pm \text { MGD013 or INCMGA00012; } \\
\text { trastuzumab+chemotherapy (XELOX or mFOLFOX-6) }\end{array}$ & Recruiting \\
\hline \multirow{3}{*}{\multicolumn{2}{|c|}{ Mavezelimab (MK-4280) }} & NCT03598608 & 1,2 & Measurable disease & Hematologic malignancies & MK-4280+pembrolizumab & Recruiting \\
\hline & & NCT02720068 & 1 & Advanced/metastatic & Solid tumors & 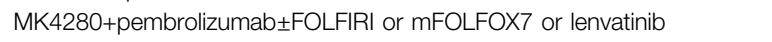 & Recruiting \\
\hline & & NCT03516981 & 2 & First line & NSCLC & MK4280+pembrolizumab or lenvatinib or quavonlimab (MK-1308) & Recruiting \\
\hline & TSR-033 & NCT03250832 & 1 & Advanced/metastatic & Solid tumors & TSR-033 \pm dostarlimab (TSR-042) \pm mFOLFOX or FOLFIRI & Recruiting \\
\hline \multirow{3}{*}{\multicolumn{2}{|c|}{ IN-CAGN02385 }} & NCT03538028 & 1 & Advanced/metastatic & Solid tumors & & Completed \\
\hline & & NCT04370704 & 1,2 & Advanced/metastatic & Solid tumors & INCAGN02385+INCAGN02390 (Anti-TIM-3)_INCMGA00012 (anti-PD-1) & Recruiting \\
\hline & & NCT03311412 & 1 & Advanced/metastatic & Solid tumors or lymphomas & Sym022+Sym021 (anti-PD-1) \pm Sym023 (anti-TIM-3) & Recruiting \\
\hline & ECO202 & NCT03600090 & 1 & Advanced/metastatic & Breast cancer & ECO202+paclitaxel & Recruiting \\
\hline & 89Zr-DFO-REGN3767 & NCT04566978 & 1 & $\begin{array}{l}\text { Measurable disease by } \\
\text { Lugano criteria }\end{array}$ & DLBCL & & Recruiting \\
\hline & $\mathrm{XmAb}^{\circledR} 22,841$ & NCT03849469 & 1 & Advanced/metastatic & Solid tumors & $\mathrm{XmAb}^{\circledR} 22841 \pm$ pembrolizumab & Recruiting \\
\hline & LBL-007 & NCT04640545 & 1 & Advanced/metastatic & Melanoma & LBL-007+toripalimab (anti-PD-1) & $\begin{array}{l}\text { Not yet } \\
\text { recruiting }\end{array}$ \\
\hline & FS118 & NCT03440437 & 1 & Advanced/metastatic & $\begin{array}{l}\text { Solid or hematologic } \\
\text { malignancy }\end{array}$ & & $\begin{array}{l}\text { Active, not } \\
\text { recruiting }\end{array}$ \\
\hline & RO7247669 & NCT04140500 & 1 & Advanced/metastatic & Solid tumors & & Recruiting \\
\hline & EMB-02 & NCT04618393 & 1,2 & Advanced/metastatic & Solid tumors & (Continued on $\mathrm{f}$ & $\begin{array}{l}\text { Not yet } \\
\text { recruiting } \\
\text { llowing page) }\end{array}$ \\
\hline
\end{tabular}


TABLE 3 | (Continued) Clinical trials on novel immune checkpoint inhibitors.

\begin{tabular}{|c|c|c|c|c|c|c|c|}
\hline Target & Drug & $\begin{array}{l}\text { Clinical } \\
\text { trial no. }\end{array}$ & Phase & Settings & Tumor types & Treatment arms & Status \\
\hline \multirow[t]{23}{*}{ TIGIT } & $\begin{array}{l}\text { Tiragolumab } \\
\text { (MTIG7192A/RG-6058) }\end{array}$ & NCT02794571 & 1 & $\begin{array}{l}\text { Locally advanced or } \\
\text { metastatic }\end{array}$ & Solid tumors & Tiragolumab \pm atezolizumab \pm chemotherapy & Recruiting \\
\hline & & NCT03563716 & 2 & $\begin{array}{l}\text { Locally advanced or } \\
\text { metastatic }\end{array}$ & NSCLC & Atezolizumab \pm tiragolumab & $\begin{array}{l}\text { Active, not } \\
\text { recruiting }\end{array}$ \\
\hline & & NCT04294810 & 3 & $\begin{array}{l}\text { Locally advanced or } \\
\text { metastatic }\end{array}$ & NSCLC & Atezolizumab \pm tiragolumab & Recruiting \\
\hline & & NCT04256421 & 3 & First line, extensive stage & SCLC & Atezolizumab+carboplatin+etoposide \pm tiragolumab & Recruiting \\
\hline & & NCT03281369 & 1,2 & Advanced/metastatic & Esophageal cancer & $\begin{array}{l}\text { Atezolizumab+tiragolumab; atezolizumab+cisplatin/5-FU士tiragolumab; } \\
\text { cisplatin/5-FU }\end{array}$ & Recruiting \\
\hline & & & & & GC, GEJ cancer & $\begin{array}{l}\text { Atezolizumab+cobimetinib with mFOFLOX6; atezolizumab+cobimetinib } \\
\text { or tiragolumab or mFOFLOX or linagliptin or PEGPH2O or BL-8040; } \\
\text { pactliaxel+ramucirumab }\end{array}$ & Recruiting \\
\hline & Vibostolimab (MK-7684) & NCT02964013 & 1 & Advanced/metastatic & Solid tumors & $\begin{array}{l}\text { Vibostolimab } \pm \text { pembrolizumab+pemetrexed/carboplatin; } \\
\text { carboplatin+cisplatin+etoposide }\end{array}$ & Recruiting \\
\hline & & NCT04305054 & 1,2 & First line & Melanoma & pembrolizumab \pm vibostolimab or quavonlimab (MK-1308) \pm lenvatinib & Recruiting \\
\hline & & NCT04305041 & 1,2 & Stage III-IV & Melanoma & pembrolizumab+quavonlimab+ vibostolimab or lenvatinib & Recruiting \\
\hline & & NCT04303169 & 1,2 & Stage III & Melanoma & pembrolizumab \pm vibostolimab or V937 (oncolytic virus) & Recruiting \\
\hline & Etigilimab (OMP-313M32) & NCT03119428 & 1 & $\begin{array}{l}\text { Locally advanced or } \\
\text { metastatic }\end{array}$ & Solid tumors & Etigilimab \pm nivolumab & Terminated \\
\hline & BMS-986207 & NCT02913313 & 1,2 & Advanced/metastatic & Solid tumors & BMS-986207 \pm nivolumab & $\begin{array}{l}\text { Active, not } \\
\text { recruiting }\end{array}$ \\
\hline & & NCT04570839 & 1,2 & Advanced/metastatic & Solid tumors & Nivolumab $\pm B M S-986207$ with COM701 (anti-PVRIG Ab) & Recruiting \\
\hline & Domvanalimab (AB-154) & NCT03628677 & 1 & Advanced/metastatic & Solid tumors & Dombvanalimab+zimberelimab (AB122, anti-PD-1) & Recruiting \\
\hline & & NCT04262856 & 2 & $\begin{array}{l}\text { Locally advanced or } \\
\text { metastatic }\end{array}$ & NSCLC & Zimberelimab \pm dombvanalimab \pm etrumadenant & Recruiting \\
\hline & ASP-8374 & NCT03945253 & 1 & Advanced/metastatic & Solid tumors & & Completed \\
\hline & & NCT03260322 & 1 & Advanced/metastatic & Solid tumors & ASP-8374 \pm pembrolizumab & $\begin{array}{l}\text { Active, not } \\
\text { recruiting }\end{array}$ \\
\hline & IBI939 & NCT04353830 & 1 & Advanced/metastatic & Solid tumors & IBI939 \pm sintilimab (anti-PD-1) & Recruiting \\
\hline & & NCT04672369 & 1 & Advanced/metastatic & NSCLC & IBI939 \pm sintilimab & $\begin{array}{l}\text { Not yet } \\
\text { recruiting }\end{array}$ \\
\hline & & NCT04672356 & 1 & Advanced/metastatic & NSCLC and SCLC & IBI939 \pm sintilimab & $\begin{array}{l}\text { Not yet } \\
\text { recruiting }\end{array}$ \\
\hline & BGB-A1217 & NCT04047862 & 1 & Advanced/metastatic & Solid tumors & BGB-A1217+tiselizumab \pm chemotherapy & Recruiting \\
\hline & COM902 & NCT04354246 & 1 & Advanced/metastatic & Solid tumors & & Recruiting \\
\hline & M6223 & NCT04457778 & 1 & Advanced/metastatic & Solid tumors & 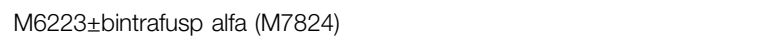 & Recruiting \\
\hline \multirow[t]{8}{*}{ TIM-3 } & Sym023 & NCT03489343 & 1 & Advanced/metastatic & Solid tumors or lymphomas & & Completed \\
\hline & LY3321367 & NCT03099109 & 1 & Advanced/metastatic & Solid tumors & LY3300054 (anti-PD-L1)+LY3321367 & $\begin{array}{l}\text { Active, not } \\
\text { recruiting }\end{array}$ \\
\hline & & NCT02791334 & 1 & Advanced/metastatic & Solid tumors & LY3300054 \pm LY3321367 or abemaciclib or ramucirumab or merestinib & $\begin{array}{l}\text { Active, not } \\
\text { recruiting }\end{array}$ \\
\hline & Cobolimab (TSR-022) & NCT02817633 & 1 & Advanced/metastatic & Solid tumors & Cobolimab \pm nivolumab or TSR-042 \pm TSR-033 \pm docetaxel & Recruiting \\
\hline & & NCT03307785 & 1 & Advanced/metastatic & Solid tumors & $\begin{array}{l}\text { Dostarlimab (TSR-042) } \pm \text { TSR-022+chemotherapy }{ }^{\mathrm{a}} \text {; } \\
\text { dostarlimab+bevacizumab } \pm \text { niraparib or chemotherapy }\end{array}$ & $\begin{array}{l}\text { Active, not } \\
\text { recruiting }\end{array}$ \\
\hline & & NCT03680508 & 2 & BCLC stage $\mathrm{B}$ or $\mathrm{C}$ & $\mathrm{HCC}$ & Cobolimab+dostarlimab & Recruiting \\
\hline & & NCT04139902 & 2 & Neoadjuvant & Melanoma & Cobolimab \pm dostarlimab & Recruiting \\
\hline & Sabatolimab (MBG453) & NCT02608268 & 1,2 & Advanced/metastatic & Solid tumors & Sabatolimab \pm spartalizumab; decitabine & $\begin{array}{l}\text { Active, not } \\
\text { recruiting }\end{array}$ \\
\hline
\end{tabular}


TABLE 3 | (Continued) Clinical trials on novel immune checkpoint inhibitors

\begin{tabular}{|c|c|c|c|c|c|c|c|}
\hline Target & Drug & $\begin{array}{l}\text { Clinical } \\
\text { trial no. }\end{array}$ & Phase & Settings & Tumor types & Treatment arms & Status \\
\hline & & NCT03961971 & 1 & Advanced/metastatic & GBM & Sabatolimab+spartalizumab & Recruiting \\
\hline & & NCT04623216 & 1,2 & $\begin{array}{l}\text { Received one prior } \\
\text { aHSCT }\end{array}$ & AML & Sabatolimab \pm azacitidine & $\begin{array}{l}\text { Not yet } \\
\text { recruiting }\end{array}$ \\
\hline & & NCT03066648 & 1 & Relapse/refractory & AML or high risk MDS & Sabatolimab \pm spartalizumab \pm decitabine & Recruiting \\
\hline & & NCT03940352 & 1 & Relapse/refractory & AML or high risk MDS & HDM201 (p53-MDM2 inhibitor)+sabatolimab or venetoclax & Recruiting \\
\hline & & NCT03946670 & 2 & $\begin{array}{l}\text { IPSS-R intermediate, high, } \\
\text { or very high risk }\end{array}$ & MDS & hypomethylating agents \pm sabatolimab & $\begin{array}{l}\text { Active, not } \\
\text { recruiting }\end{array}$ \\
\hline & & NCT04266301 & 3 & $\begin{array}{l}\text { IPSS-R intermediate, high, } \\
\text { or very high risk for MDS }\end{array}$ & MDS or $\mathrm{CML}$ & Sabatolimab+azacitidine & Recruiting \\
\hline & INCAGN2390 & NCT03652077 & 1 & Advanced/metastatic & Solid tumors & & $\begin{array}{l}\text { Active, not } \\
\text { recruiting }\end{array}$ \\
\hline & BMS-986258 & NCT03446040 & 1,2 & Advanced/metastatic & Solid tumors & BMS-986258+nivolumab or rHuPH2O & Recruiting \\
\hline & SHR-1702 & NCT03871855 & 1 & Advanced/metastatic & Solid tumors & 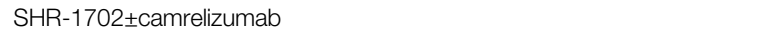 & Unknown \\
\hline & RO7121661 & NCT03708328 & 1 & Advanced/metastatic & Solid tumors & & Recruiting \\
\hline \multirow[t]{22}{*}{ B7-H3 } & Enoblituzumab (MGA271) & NCT01391143 & 1 & Advanced/metastatic & Solid tumors & & Completed \\
\hline & & NCT02982941 & 1 & Advanced/metastatic & Pediatric solid tumors & & Completed \\
\hline & & NCT02923180 & 2 & $\begin{array}{l}\text { Localized intermediate } \\
\text { and high-risk }\end{array}$ & Prostate cancer & & $\begin{array}{l}\text { Active, not } \\
\text { recruiting }\end{array}$ \\
\hline & & NCT04634825 & 2 & Advanced/metastatic & HNSCC & $\begin{array}{l}\text { Enoblituzumab+retifanlimab (anti-PD-1 antibody) or tebotelimab (PD-1 } \\
\text { and LAG-3 bispecific DART molecule) }\end{array}$ & $\begin{array}{l}\text { Not yet } \\
\text { recruiting }\end{array}$ \\
\hline & & NCT02381314 & 1 & Advanced/metastatic & Solid tumors & Enoblituzumab+ipilimumab & Completed \\
\hline & & NCT02475213 & 1 & Advanced/metastatic & Solid tumors & Enoblituzumab+pembrolizumab or retifanlimab & $\begin{array}{l}\text { Active, not } \\
\text { recruiting }\end{array}$ \\
\hline & & NCT04129320 & 2,3 & Advanced/metastatic & HNSCC & Enoblituzumab+retifanlimab or tebotelimab & Withdrawn \\
\hline & DS-7300a & NCT04145622 & 1,2 & Advanced/metastatic & Solid tumors & & Recruiting \\
\hline & Orlotamab (MGD009) & NCT02628535 & 1 & Advanced/metastatic & solid tumors & & Terminated \\
\hline & & NCT03406949 & 1 & Advanced/metastatic & Solid tumors & Orlotamab+retifanlimab & $\begin{array}{l}\text { Active, not } \\
\text { recruiting }\end{array}$ \\
\hline & 1311-Omburtamab & NCT01099644 & 1 & Peritoneal involvement & DSRCT & & $\begin{array}{l}\text { Active, not } \\
\text { recruiting }\end{array}$ \\
\hline & & NCT00089245 & 1 & Advanced/metastatic & $\begin{array}{l}\text { CNS or leptomeningeal } \\
\text { cancer }\end{array}$ & & $\begin{array}{l}\text { Active, not } \\
\text { recruiting }\end{array}$ \\
\hline & & NCT03275402 & 2,3 & Recurrent & $\begin{array}{l}\text { Neuroblastoma, CNS, or } \\
\text { leptomeningeal metastases }\end{array}$ & & Recruiting \\
\hline & 124I-Omburtamab & NCT01502917 & 1 & $\begin{array}{l}\text { Prior external beam } \\
\text { radiotherapy }\end{array}$ & Gliomas & 124I-Omburtamab+external beam radiotherapy (prior to study entry) & Recruiting \\
\hline & $\begin{array}{l}\text { 177Lu-DTPA- } \\
\text { Omburtamab }\end{array}$ & NCT04167618 & 1,2 & Recurrent & Medulloblastoma & & $\begin{array}{l}\text { Not yet } \\
\text { recruiting }\end{array}$ \\
\hline & & NCT04315246 & 1,2 & Advanced/metastatic & $\begin{array}{l}\text { Leptomeningeal metastasis } \\
\text { from solid tumors }\end{array}$ & & $\begin{array}{l}\text { Not yet } \\
\text { recruiting }\end{array}$ \\
\hline & 4SCAR-276 & NCT04432649 & 1 & Advanced/metastatic & Solid tumors & & Recruiting \\
\hline & SCRI-CARB7H3 & NCT04185038 & 1 & Advanced/metastatic & Pediatric CNS tumors & & Recruiting \\
\hline & B7-H3 CAR-T & NCT04385173 & 1 & Recurrent & GBM & B7-H3 CAR-T+temozolomide & Recruiting \\
\hline & & NCT04077866 & 1,2 & Recurrent & GBM & B7-H3 CAR-T \pm temozolomide & Recruiting \\
\hline & CAR.B7-H3 & NCT04670068 & 1 & Advanced/metastatic & Epithelial ovarian cancer & B7-H3 CAR-T+fludarabine+cyclophosphamide & $\begin{array}{l}\text { Not yet } \\
\text { recruiting }\end{array}$ \\
\hline & $\begin{array}{l}\text { Second generation 4- } \\
\text { 1BB } \zeta \text { B } 7 \text { H3-EGFRt-DHFR }\end{array}$ & NCT04483778 & 1 & Recurrent & $\begin{array}{l}\text { Non-primary CNS solid } \\
\text { tumors }\end{array}$ & 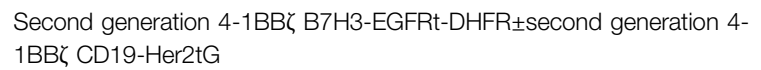 & Recruiting \\
\hline
\end{tabular}


TABLE 3 | (Continued) Clinical trials on novel immune checkpoint inhibitors.

\begin{tabular}{|c|c|c|c|c|c|c|c|}
\hline Target & Drug & $\begin{array}{l}\text { Clinical } \\
\text { trial no. }\end{array}$ & Phase & Settings & Tumor types & Treatment arms & Status \\
\hline \multirow[t]{3}{*}{ VISTA } & JNJ-61610588 & NCT02671955 & 1 & Advanced/metastatic & Solid tumors & & Terminated \\
\hline & $\mathrm{Cl}-8993$ & NCT04475523 & 1 & Advanced/metastatic & Solid tumors & & Recruiting \\
\hline & CA-170 & NCT02812875 & 1 & Advanced/metastatic & Solid tumors or lymphomas & & Completed \\
\hline \multirow[t]{6}{*}{ Icos } & GSK3359609 & NCT04428333 & 1,2 & Advanced/metastatic & HNSCC & GSK3359609 \pm pembrolizumab+fluouracil-platinum based chemotherapy & Recruiting \\
\hline & & NCT04128696 & 3 & Advanced/metastatic & HNSCC & GSK3359609+pembrolizumab & Recruiting \\
\hline & & NCT03693612 & 2 & Advanced/metastatic & Solid tumors & GSK3359609+pembrolizumab; docetaxel+paclitaxel+cetuximab & Recruiting \\
\hline & JTX-2011 & NCT02904226 & 1,2 & Advanced/metastatic & Solid tumors & JTX-2011+pembrolizumab or nivolumab or ipilimumab & Completed \\
\hline & MEDI-570 & NCT02520791 & 1 & Advanced/metastatic & Lymphoma & & Recruiting \\
\hline & KY1044 & NCT03829501 & 1,2 & Advanced/metastatic & Solid tumors & KY1044_atezolizumab & Recruiting \\
\hline \multirow[t]{6}{*}{ BTLA } & INBRX-106 & NCT04198766 & 1 & $\begin{array}{l}\text { Locally advanced or } \\
\text { metastatic }\end{array}$ & Solid tumors & INBRX-106+pembrolizumab & Recruiting \\
\hline & Cudarolimab (IB|101) & NCT03758001 & 1 & Advanced/metastatic & Solid tumors & Cudarolimab+sintilimab (anti-PD-1) & Recruiting \\
\hline & PF-04518600 & NCT02315066 & 1 & Advanced/metastatic & Solid tumors & PF-04518600ะutomilumab (PF-05082566, anti-TNFRSF9) & Completed \\
\hline & TAB004 (JS004) & NCT04137900 & 1 & Advanced/metastatic & Solid tumors or lymphomas & & Recruiting \\
\hline & & NCT04278859 & 1 & Advanced/metastatic & Solid tumors & & Recruiting \\
\hline & & NCT04477772 & 1 & Advanced/metastatic & Lymphoma & & Recruiting \\
\hline
\end{tabular}

Abbreviations: AML, acute myeloid leukemia; anti-PD-1, anti-programmed death-1; BCLC, Barcelona Clinic Liver Stage; BTLA, B and T-lymphocyte attenuator; CML, chronic myelogenous leukemia; CNS, central nervous system; CRC, colorectal cancer; DART, dual-affinity re-targeting proteins; DLBCL, diffuse large B cell lymphoma; DSRCT, desmoplastic small round cell tumor; GBM, glioblastoma multiforme; GC, gastric cancer; GEJ, gastroesophageal junction cancer; HCC, hepatocellular carcinoma; HNSCC, head and neck squamous cell carcinoma; ICOS, Inducible T cell costimulator; IDO1;, indoleamine 2,3-dioxygenase-1 inhibitor; IPSS-R, revised international prognostic scoring system; LAG3, lymphocyte-associated gene 3; MDM2, mouse double minute 2 homolog; MDS, myelodysplastic syndrome; MSI-H, microsatellite instability-high; MSS, microsatellite stable; NSCLC, non-small cell lung cancer; PEGPH2O, pegylated recombinant human hyaluronidase; RCC, Renal cell carcinoma; rHuPH2O, recombinant human hyaluronidase PH2O enzyme; SCLC, small cell lung carcinoma; TIGIT, T cell immunoglobulin and ITIM domain; TIM, T-cell immunoglobulin and mucin domain-3; TNBC, triple negative breast cancer; TNFRSF9, tumor necrosis factor receptor superfamily member 9; VEGF, vascular endothelial growth factor; VISTA, V-domain immunoglobulin suppressor of T cell activation. Regimens: $\mathrm{mFOLFOX,} \mathrm{oxaliplatin} 85 \mathrm{mg} / \mathrm{m}^{2}$ intravenous (IV), leucovorin $400 \mathrm{mg} / \mathrm{m}^{2} \mathrm{IV}$, and fluorouracil $2400 \mathrm{mg} / \mathrm{m}^{2} \mathrm{IV} \mathrm{over} 46-48$ h every 2 weeks (Q2W) FOLFIRl, irinotecan $180 \mathrm{mg} / \mathrm{m}^{2} \mathrm{IV}$, leucovorin $400 \mathrm{mg} / \mathrm{m}^{2} \mathrm{IV}$, and 5-FU $2400 \mathrm{mg} /$ $m^{2}$ IN over 46-48 h (Q2W)

${ }^{a}$ Chemotherapy: carboplatin/pemetrexed, carboplatin/nab-paclitaxel, or carboplatin/paclitaxel. 
Recently, several studies have highlighted that TIGIT is co-expressed and associated with PD-1 expression (Johnston et al., 2014; Chauvin et al., 2015). Dual blockade of TIGIT and $\mathrm{PD}-1$ resulted in the restoration of T-cell immunity in preclinical settings and provided a rationale for combination with these agents as a feasible anti-cancer therapeutic strategy (Johnston et al., 2014; Kurtulus et al., 2015; Zhang et al., 2018).

\section{Clinical Trials on TIGIT}

Among the 10 anti-TIGIT mAbs undergoing clinical trials, one of the most promising agents is tiragolumab (GO30103) (Table 2). In a randomized, double-blind, phase 2 trial, 135 treatment-naïve patients with unresectable and metastatic NSCLC, positive for PD-L1 expression, were treated with tiragolumab (or placebo) in combination with atezolizumab (anti-PD-L1) (NCT03563716) (Rodriguez-Abreu et al., 2020). Primary analysis of CITYSCAPE showed that the result was significant and durable, especially in patients with a PD-L1 tumor proportion score (TPS) $\geq 50 \%$ in the tiragolumab and atezolizumab groups, with an ORR of 31.3 vs. 16.2\% and median PFS of 5.4 and 3.6 months in the combination treatment and atezolizumab monotherapy, respectively (hazard ratio $0.57,95 \%$ confidence interval [CI] 0.37-0.90). The combination was well tolerated and had acceptable safety profiles. The positive and robust results of this trial prompted initiation of phase III in select patients with high PD-L1 expression (SKYSCRAPER-1, NCT04294810). Furthermore, the combination was supplemented with chemotherapy in chemotherapy-naive extensive stage SCLC (SKYSCRAPER-2, NCT04256421). Phase 1 and 2 clinical trials on tiragolumab are also ongoing for esophageal and gastric cancers (NCT03281369) in metastatic settings.

Vibostolimab (MK-7684) is also an anti-TIGIT mAb. The preliminary results of a phase 1 dose-finding study of vibostolimab (200 or $210 \mathrm{mg}$ ) with pembrolizumab (200 mg) on day 1 of each Q3W cycle administered to patients with advanced/metastatic solid tumors without prior anti-PD-1/PD-1, showed acceptable toxicity profiles (NCT02964013) (Niu et al., 2020). The ORR and median PFS were $29 \%$ and 5.4 months for all patients, and $46 \%$ and 8.4 months for 13 patients with TPS $\geq 1 \%$, respectively. The effects of vibostolimab are also being investigated in melanoma, in combination with other agents (NCT04305054, NCT04305041, and NCT04303169).

Other anti-TIGIT mAbs under investigation include BMS986207 (NCT02913313 and NCT04570839), domvanalimab (AB-154) (NCT03628677 and NCT04262856), ASP-8374 (NCT03945253 and NCT03260322), IBI939 (NCT04353830, NCT04672369, and NCT04672356), BGB-A1217 (NCT04047862), COM902 (NCT04354246), and M6223 (NCT04457778) as monotherapy or in combination with other agents in the treatment of refractory solid tumors. These agents are being tested in phase $1 / 2$ trials and the results are awaited.

\section{TIM-3}

TIM-3, previously known as hepatitis A virus cellular receptor 2 (HAVCR2), is a member of the TIM gene family, encoding proteins such as TIM-1 and TIM-4 (Table 1) (Monney et al., 2002). It is structured with type- 1 cell surface glycoproteins, an extracellular Ig variable region ( $\mathrm{IgV}$ )-like domain, a mucin-like and transmembrane domain, and an intracellular cytoplasmic tail composed of five tyrosine residues (Monney et al., 2002). Once the two tyrosine residues, Y265 and 272, are phosphorylated by Src kinases or interleukin inducible T cell kinase, the downstream signaling of TIM-3 is activated (van de Weyer et al., 2006; Nagahara et al., 2008).

TIM-3 is expressed in tumor cells and immune cells, such as helper T cells (Th1), IL-17-producing $\mathrm{CD}^{+}$effector cell lineage (Th17), $\mathrm{CD}^{+} \mathrm{T}$ cells, Tregs, TILs, and innate immune cells (Monney et al., 2002; Huang et al., 2010; Jan et al., 2011; Anderson, 2012). Four ligands bind to TIM-3: two soluble ligands, high-mobility group protein B1 (HMGB1) and galectin-9, and two surface ligands, including carcinoembryonic antigen cell adhesion molecule 1 (ceacam-1) and phosphatidyl serine (PtdSer) (Zhu et al., 2005; Nakayama et al., 2009; Chiba et al., 2012; Huang et al., 2015; Kang et al., 2015). Interaction of TIM-3 with its ligands has been shown to induce T cell inhibition. TIM-3 is unique compared to other immune checkpoints in that its upregulation is initiated only by $\mathrm{CD} 4^{+}$and $\mathrm{CD}^{+}$cells that produce IFN- $\gamma$ (Sakuishi et al., 2010; Gao et al., 2012).

Similar to PD-L1, TIM-3 is expressed in TILs is associated with disease progression in certain cancers (Ngiow et al., 2011). Meta-analysis of TIM-3 overexpression in solid tumors has shown that higher TIM-3 expression is associated with worse $O S$ and may potentially be a prognostic marker (Zhang et al., 2017). Blocking TIM-3 expression results in $\mathrm{T}$ cell proliferation and cytokine production, thereby eliciting immune activation (Gao et al., 2012). In addition, targeting TIM-3 with PD-1 in preclinical settings has shown a synergistic effect by reinvigorating $\mathrm{T}$ cell function and increasing anti-tumor immunity (Sakuishi et al., 2010; Koyama et al., 2016). Thus, the dual blockade of PD-1 and TIM-3 is a feasible and promising therapeutic option.

\section{Clinical Trials on TIM-3}

There are seven anti-TIM-3 mAbs and one anti-PD-1 and TIM-3 bispecific Ab (RO7121661) undergoing clinical trials (Table 2). Sym021 (anti-PD-1), sym022 (anti-LAG-3), and sym023 (anti-TIM-3) were evaluated as single agents or combinations in phase 1 trials for solid tumors or lymphomas (NCT03311412, NCT03489369, and NCT03489343) (Lakhani et al., 2020). Sym023 monotherapy $(n=24)$ and in combination with Sym021 $(n=17)$ was administered; however, Sym023 and its combination did not reach their MTD. One patient in the monotherapy group had grade 3-4 immune-mediated arthritis. Overall, monotherapy and combination therapy were well tolerated, with two PRs observed in the combination group.

LY3321367 is also an anti-TIM-3 mAb; an interim analysis of a phase $1 \mathrm{a} / 1 \mathrm{~b}$, dose-escalation and -expansion study showed that intravenous infusion of 3-1200 mg LY3321367 Q2W monotherapy (Arm A, 23 patients) or 70-1200 mg LY3321367 
+ 200-700 mg LY3300054 (anti-PD-L1) Q2W combination therapy (Arm B, 18 patients) was well tolerated in the treatment of refractory solid tumors; further, no DLT was observed and most TRAEs observed were grade $\leq 2$ (NCT03099109) (Harding et al., 2019). Two patients in arm A showed $>20 \%$ tumor reduction. Overall, there was no effect on the pharmacokinetics, and the antidrug antibody titers were low; thus, Eli Lily dropped the agent from its pipeline.

Other investigational agents targeting TIM-3 include cobolimab (TSR-022), sabatolimab, INCAGN2390, BMS986258, SHR-1702, and RO7121661, which are currently ongoing clinical trials. Cobolimab is administered in combination with chemotherapy, targeted agents, or immune checkpoints in solid tumors (NCT02817633, NCT03307785, NCT03680508, and NCT04139902). Sabatolimab (MBG453) is administered with other agents in solid tumors (NCT02608268 and NCT03961971) or in acute myeloid lymphoma (AML) (NCT04623216), high-risk myelodysplastic syndrome (MDS) (NCT03066648, NCT03940352, and NCT03946670), and chronic myelogenous leukemia (CML) (NCT04266301). In solid tumors, INCAGN2390 is administered as a monotherapy (NCT03652077), BMS-986258 is administered in combination with nivolumab or rHuPH20 (NCT03446040), SHR-1702 is administered with or without camrelizumab, an anti-PD-1 agent (NCT03871855), and RO7121661, a TIM-3 bispecific $\mathrm{Ab}$, is administered as a monotherapy (NCT03708328).

\section{B7-H3}

B7-H3, also called CD276, is a member of the B7 family. It was initially recognized as a co-stimulatory molecule that activates T cells and IFN- $\gamma$ production (Table 1) (Chapoval et al., 2001). $\mathrm{B} 7-\mathrm{H} 3$ is found in activated immune cells such as antigenpresenting cells (APCs), NK cells, T cells, and monocytes (Janakiram et al., 2017). In addition, B7-H3 is expressed in several tumors. Notably, high levels of $\mathrm{B} 7-\mathrm{H} 3$ expression in NSCLC, RCC, CRC, and prostate cancer are correlated with disease progression (Li et al., 2014; Jin et al., 2015; Benzon et al., 2017; Mao et al., 2017). In NSCLC, B7-H3 with Tregs was associated with poor prognosis, and co-expression of B7-H3 and CD14 was found to play a role in angiogenesis and tumor progression in RCC (Li et al., 2014; Jin et al., 2015). Patients with $\mathrm{CRC}$, harboring $\mathrm{B} 7-\mathrm{H} 3$ and $\mathrm{CD} 133$ expression, have shorter survival (Castellanos et al., 2017). Similarly, high levels of $\mathrm{B} 7-\mathrm{H} 3$ are associated with higher Gleason grade, advanced stage, and poor outcomes in prostate cancer (Benzon et al., 2017).

Recently, the co-inhibitory function of B7-H3 in $\mathrm{CD}^{+}$and $\mathrm{CD}^{+} \mathrm{T}$ cells was discovered (Suh et al., 2003; Prasad et al., 2004). Studies are ongoing to identify the receptor for B7-H3, and the contradictory roles of B7-H3 in immune activity are yet to be fully elucidated (Yang et al., 2020). In addition to the immunological aspects of $\mathrm{B} 7-\mathrm{H} 3$, other signaling pathways, including PI3K/AKT/ mTOR, JAK2/STAT3, and TLR4/NF- $\kappa B$ signaling, can activate B7H3 expression (Kang et al., 2015; Zhang et al., 2015; Xie et al., 2016; Fan et al., 2017; Zhang et al., 2017). Other studies have highlighted that B7-H3 is associated with resistance to chemotherapy and targeted agents (Liu et al., 2011; Jiang et al., 2016; Flem-Karlsen et al., 2017; Flem-Karlsen et al., 2019).

\section{Clinical Trials on B7-H3}

Eleven agents targeting B7-H3 are currently under investigation in clinical trials (Table 2). Generally, patients harboring B7-H3 are enrolled in clinical trials. Enoblituzumab (MGA271), an antiB7-H3 mAb with antibody-dependent cellular toxicity (ADCC) function, has been investigated in multiple solid tumors, including pediatric tumors. Interim analysis of enoblituzumab in refractory solid tumors revealed that it was well tolerated up to $15 \mathrm{mg} / \mathrm{kg}$, with no DLT and MTD (Powderly et al., 2015). Although TRAEs, such as fatigue $(30 \%)$ and infusion-related reactions $(26 \%)$, occurred in $71 \%$ of the patients, most of these AEs were tolerated with adequate supportive care (NCT01391143). Enoblituzumab is currently being used as a monotherapy or in combination with anti-PD-1 antibody (retifanlimab or pembrolizumab), tebotelimab, a PD-1 and LAG-3 bispecific DART, or ipilimumab, as shown in Table 3.

DS-7300a is a B7-H3-targeting antibody drug conjugate (ADC) with $\mathrm{DXd}$, a payload that is an exatecan derivative, which inhibits topoisomerase I (Bendell et al., 2020). The phase 1/II study is ongoing with patients enrolled in the doseescalation part (NCT04145622). Orlotamab (MGD009) is a B7$\mathrm{H} 3$ and $\mathrm{CD} 3$ DART protein, and its monotherapy (NCT02628535) and combination with retifanlimab (NCT03406949) are under investigation in heavily treated solid tumors. Orlotamab with radioactive labeling such as 131I-Omburtamab (NCT01099644, NCT00089245, and NCT03275402), 124I-Omburtamab (NCT01502917), and 177Lu-DTPA-Omburtamab (NCT04167618 and NCT04315246) are also ongoing trials. In patients with desmoplastic small round cell tumor (DSRCT), treatment with 131I-Omburtamab via intraperitoneal administration followed by external beam intensity-modulated whole-abdominopelvic radiotherapy (WAP-IMRT) to 3,000 cGy was tolerable with a satisfactory safety profile, and appeared to demonstrate micrometastatic activity in a phase 1 trial (Modak et al., 2018). The biodistribution, organ, and whole-body exposure were measured with 124I-8H9-directed radioimmuno-PET, and the RP2D for 131I-Omburtamab was set at $80 \mathrm{mCi} / \mathrm{m}^{2}$.

Other investigational agents include chimeric antigen receptor (CAR) $\mathrm{T}$ cell therapy targeting B7-H3: 4SCAR-276 in solid tumors (NCT04432649), SCRI-CARB7H3 in pediatric CNS tumors (NCT04185038), B7-H3 chimeric antigen receptor $\mathrm{T}$ cells (CAR-T) treated alone (NCT04385173) or with temozolamide (NCT04077866) in glioblastoma, CAR.B7-H3 with other agents in epithelial ovarian cancer (NCT04670068), and second-generation $4-1 \mathrm{BB} \zeta \mathrm{B} 7 \mathrm{H} 3$-EGFRt-DHFR in nonprimary CNS solid tumors (NCT04483778).

\section{VISTA}

VISTA has several names such as differentiation of embryonic stem cells 1 (Dies1), DD1 a, Gi24, and B7H5 (Table 1). (Ceeraz 
et al., 2013). Notably, it is also named PD-1 homologue (PD$1 \mathrm{H})$, as its extracellular domain shows structural similarity to PD-1; however, it is different, as it lacks the classical ITIM or ITSM motif in the cytoplasmic domain (Flies et al., 2011). Furthermore, VISTA differs from PD-1, which functions in the effector stage, as VISTA is expressed on resting $\mathrm{T}$ cells, indicating its regulatory role in earlier stages (Kondo et al., 2016). Compared to that in peripheral lymph nodes, VISTA is more abundant in myeloid-derived suppressor cells (MDSCs) in the tumor microenvironment (TME) (Le Mercier et al., 2014).

High levels of VISTA are expressed by mature APCs with $\mathrm{CD} 11 \mathrm{~b}$, whereas relatively low expression is found on Tregs, $\mathrm{CD}^{+}, \mathrm{CD}^{+}$, and TILs (Lines et al., 2014). Although the counter structures for VISTA have not been comprehensively elucidated, recent in vitro findings on $\mathrm{V}$-Set and immunoglobulin domain containing 3 (VSIG-3) have shown that VISTA also acts as a coinhibitory ligand on tumor cells (Wang et al., 2019). VISTA promotes Treg maturation and prevents $\mathrm{T}$ cell activation independent of PD-1 expression (Yoon et al., 2015; Torphy et al., 2017; Popovic et al., 2018). The non-overlapping mechanisms of VISTA and PD-L1 make their combination an ideal treatment strategy to overcome immune suppression. In mouse models, dual blockade of VISTA and PD-1, using monoclonal antibodies specific for these immune checkpoints, led to synergistic activity against T-cells with anti-tumor responses (Liu et al., 2015).

A wide array of tumors has been studied to determine the prognostic and predictive roles of VISTA. High-grade serous ovarian cancer patients with tumor cells expressing VISTA showed longer PFS and OS (Zong et al., 2020). Furthermore, VISTA expression on TILs in pT1/2 esophageal adenocarcinoma was associated with improved OS compared to the TILs negative for VISTA (Loeser et al., 2019). Similarly, VISTA $^{+}$and $\mathrm{CD}^{+}$TIL subtypes are associated with better OS in HCC (Zhang et al., 2018). Contrary to these findings, $\mathrm{VISTA}^{+}$and $\mathrm{CD} 8^{+}$TIL subtypes were associated with worse prognosis in oral squamous cell carcinoma and cutaneous melanoma with VISTA expression, whereas VISTA had no correlation with survival outcome in GC expressing VISTA(Böger et al., 2017; Wu et al., 2017; Kuklinski et al., 2018).

\section{Clinical Trials on VISTA}

Ongoing clinical trials on VISTA include two anti-VISTA $\mathrm{mAbs}$ and one small-molecule antagonist of VISTA (Table 2). JNJ-61610588 (NCT02671955) and CI-8993 (NCT04475523) are anti-VISTA mAbs, currently under investigation in phase 1 trials for the treatment of refractory solid tumors. CA-170 is a small molecule that targets both VISTA and PD-L1 (Musielak et al., 2019). A phase 1 study in patients with advanced solid tumors or lymphomas showed no DLT during dose escalation in 19 patients treated across six dose levels (50-800 mg) (NCT02812875) (Powderly et al., 2017). Exploratory analysis showed an increased proportion of both circulating $\mathrm{CD}^{+}$and $\mathrm{CD}^{+}$cells after oral dosing with CA-170. Further data on dose escalation, the recommended phase 2 dose, and anti-tumor responses are awaiting results.

\section{ICOS}

ICOS, also known as cluster of differentiation 278 (CD278) in $\mathrm{T}$ cells, is a member of the CD28 coreceptor family, which includes costimulatory CD28 and coinhibitory receptor CTLA-4 (Table 1) (Hutloff et al., 1999). The ICOS ligand (ICOSL) is expressed in APCs such as macrophages, DCs, and B cells (Yoshinaga et al., 1999). In contrast to the expression of CD28 in both naive and memory T cells, the majority of ICOS is expressed only after the activation of memory $\mathrm{T}$ cells, with only small fractions expressed in resting memory $\mathrm{T}$ cells. Further, unlike CD28 and CTLA-4 ligands, which are expressed primarily on lymphoid tissues, ICOSL is expressed in non-lymphoid cells, such as endothelial cells, epithelial cells, mesenchymal cells, and fibroblasts, via the activation of tumor necrosis factor-a (Swallow et al., 1999; Khayyamian et al., 2002; Martin-Orozco et al., 2010). Activation of the ICOS pathway induces the production of cytokines, such as IL-4, IL-10, and IL-21, by CD $4^{+}$Th cells, $\mathrm{CD}^{+}$forkhead box $\mathrm{P} 3\left(\mathrm{FoxP}^{+}\right.$) Tregs, and $\mathrm{CD} 8^{+}$cytotoxic $\mathrm{T}$ lymphocytes (CTL) (Hutloff et al., 1999; Gigoux et al., 2009; Solinas et al., 2020). ICOS interacts with its ligand (ICOSL) to increase anti-tumor effects via the regulation of memory and effector $\mathrm{T}$ cell development and humoral immune responses (Marinelli et al., 2018). The rationale for targeting the ICOS/ ICOSL axis with agonists and antagonists is its capacity to trigger both anti-tumor $\mathrm{T}$ cell responses by $\mathrm{Th} 1$ and other effector T cells, as well as its protumor responses via Tregs (Solinas et al., 2020).

In preclinical studies, ICOS expression on FoxP3 ${ }^{+}$Tregs and other Th subsets has been identified in multiple arrays of solid tumors, including melanoma, gastric, colorectal, and breast cancers (Strauss et al., 2008; Zhang et al., 2016; Gu-Trantien et al., 2017; Nagase et al., 2017). ICOS ${ }^{+}$Treg TILs have been found to be associated with worse survival in GC, whereas high levels of ICOS in Th1 TILs in colorectal cancer indicated better survival outcomes (Zhang et al., 2016; Nagase et al., 2017). Dual blockade of ICOS with anti-CTLA-4 has been effective in eliciting anti-tumor responses in ICOS knockout mice that were unresponsive to anti-CTLA-4 monotherapy (Fu et al., 2011; Fan et al., 2014). More importantly, the utilization of ICOStargeted agents is gaining attention in hematological malignancies owing to the enhancement of co-stimulatory receptor $4-1 \mathrm{BB}$ in $\mathrm{CD}^{+}$CAR $\mathrm{T}$ cells by ICOS (Guedan et al., 2018).

\section{Clinical Trials on ICOS}

Currently, both anti-ICOS agonists and anti-ICOS antagonists are under clinical investigation (Table 2). The phase 1 trial of GSK3359609 (INDUCE-1), a humanized antiICOS agonist monoclonal antibody, comprised two treatment groups: part 1 patients were treated with a monotherapy of GSK3359609, and part 2 patients were 
administered a combination with pembrolizumab or other immunotherapy in the treatment of advanced solid tumors. The study is ongoing, with no dose-limiting toxicities from the first three dose-limiting cohorts (Angevin et al., 2017). In head and neck cancer, the efficacy of GSK3359609 and pembrolizumab with or without platinum-based chemotherapy is currently under investigation (NCT04428333 and NCT04128696).

Another investigational anti-ICOS agonist monoclonal antibody is JTX-2011, used in combination with either antiPD1 (pembrolizumab or nivolumab) or anti-CTLA-4 (ipilimumab) in advanced solid tumors (NCT02904226) (Yap et al., 2018). In phase $1 /$ II of the trial, anti-tumor activity was observed with JTX-2011 monotherapy and in combination with nivolumab, in heavily treated GC and TNBC with manageable toxicity profiles. Exploratory analysis showed that the peripheral blood CD4 ICOS ${ }^{\text {high }} \mathrm{T}$ cell subsets may be a potential biomarker for the response.

Further, agonistic antibodies such as MEDI-570 alone and KY1044 with atezolizumab are under investigation in phases 1 and phase 1/II, respectively (NCT02520791 and NCT03829501).

\section{BTLA}

BTLA (CD272) is also a member of the CD28 coreceptor family (Table 1) (Ceeraz et al., 2013). It is a co-inhibitory molecule with a structure and function similar to those of PD-1 and CTLA-4 (Paulos and June, 2010). When expressed on mature lymphocytes, such as $\mathrm{B}$ cells and $\mathrm{T}$ cells, macrophages, and DCs, BTLA binds to herpes virus entry mediator (HVEM), a member of the tumor necrosis factor receptor superfamily (TNFRSF), as well as to LIGHT and lymphotoxin- $\alpha$, two members of the tumor necrosis factor (TNF) superfamily (Han et al., 2004; Sedy et al., 2005; Steinberg et al., 2011). Binding of BTLA to HVEM via CD160 transmits inhibitory signals to $\mathrm{T}$ cells, which are necessary for proliferation and cytokine production, whereas binding to LIGHT induces co-stimulatory signals (Sedy et al., 2005; Murphy et al., 2006; Cai et al., 2008). Thus, the complexity of the BTLA receptor and ligand activity poses a challenge for BTLA blockade treatment.

Recently, the possibility of BTLA as a potential therapeutic target in cancer immunotherapy has been established in vivo, wherein human melanoma tumor antigen-specific effector $\mathrm{CD}^{+} \mathrm{T}$ cells expressing high levels of BTLA were downregulated with a vaccine formulated using $\mathrm{CpG}$ oligodeoxynucleotides, a toll-like receptor 9 (TLR9) agonist that triggers innate immunity, thereby proving that inhibition of BTLA may partially reverse the function of human $\mathrm{CD}^{+}$

\section{REFERENCES}

Anderson, A. C., Joller, N., and Kuchroo, V. K. (2016). Lag-3, Tim-3, and TIGIT: Co-inhibitory Receptors with Specialized Functions in Immune Regulation. Immunity 44, 989-1004. doi:10.1016/j.immuni.2016.05.001 cancer-specific T cells (Derré et al., 2010; Paulos and June, 2010).

\section{Clinical Trials on BTLA}

There are four agents targeting BTLA (Table 2): 1) INBRX106, a hexavalent OX40 agonist Ab (NCT04198766), 2) PF04518600 (NCT02315066), an OX40 agonist; 3) cudarolimab (IBI101) (NCT03758001), an anti-OX40 $\mathrm{mAb}$, and 4) TAB004 (JS004) (NCT04278859), an antiBTLA mAb. These agents target the OX40 receptor, also known as CD134 and tumor necrosis factor receptor superfamily member 4 (TNFRSF4), thereby preventing its interaction with BTLA (Croft et al., 2009). These phase 1 clinical trials are ongoing as monotherapy for patients with advanced/metastatic solid tumors and are awaiting results. TAB004 is also under investigation for the treatment of refractory lymphomas (NCT04137900 and NCT04477772).

\section{CONCLUSION}

Cancer immunotherapy is one of the major pillars in the field of medical oncology, especially for the treatment of unresectable, metastatic, and recurrent cancers. The success of ICIs, such as antiCTLA-4 and anti-PD-1/PD-L1, in combination with chemotherapy, immunotherapy, and targeted agents, has changed the paradigm of cancer treatment. Nonetheless, the limited efficacy and IRAEs of ICIs have paved way for the discovery of novel checkpoints. Among the immune checkpoint inhibitors, anti-LAG-3 and anti-TIGIT are promising targets, and their efficacy in combination with anti-PD1/PD-L1 may help overcome the limitations seen in prior treatments. More robust data are yet to follow on agents targeting TIM-3, B7-H3, VISTA, ICOS, and BTLA.

\section{AUTHOR CONTRIBUTIONS}

All authors listed have made a substantial, direct, and intellectual contribution to the work and approved it for publication.

\section{FUNDING}

This work was supported by National Research Foundation of Korea (NRF) grants funded by the Korean Government (MSIT) (NRF-2017M3A9E9072669, 2017M3A9E8029717, NRF-2019M3A9B6065231, 2019M3A9B6065221, 2018R1A2A1A05076997, 2017R1A5A1014560).

Anderson, A. C. (2012). Tim-3, a Negative Regulator of Anti-tumor Immunity. Curr. Opin. Immunol. 24, 213-216. doi:10.1016/j.coi.2011.12.005

Andreae, S., Piras, F., Burdin, N., and Triebel, F. (2002). Maturation and Activation of Dendritic Cells Induced by Lymphocyte Activation Gene-3 (CD223). J. Immunol. 168, 3874-3880. doi:10.4049/jimmunol.168.8. 3874 
Andrews, L. P., Marciscano, A. E., Drake, C. G., and Vignali, D. A. (2017). LAG3 (CD223) as a Cancer Immunotherapy Target. Immunol. Rev. 276, 80-96. doi:10.1111/imr.12519

Angevin, E., Barnette, M. S., Bauer, T. M., Cho, D. C., Ellis, C. E., Gan, H. K., et al. (2017). INDUCE-1: A Phase I Open-Label Study of GSK3359609, an ICOS Agonist Antibody, Administered Alone and in Combination with Pembrolizumab in Patients with Advanced Solid Tumors. Jco 35, TPS3113. doi:10.1200/JCO.2017.35.15_suppl.TPS3113

Atkinson, V., Khattak, A., Haydon, A., Eastgate, M., Roy, A., Prithviraj, P., et al. (2020). Eftilagimod Alpha, a Soluble Lymphocyte Activation Gene-3 (LAG-3) Protein Plus Pembrolizumab in Patients with Metastatic Melanoma. J. Immunother. Cancer 8, e001681. doi:10.1136/jitc-2020-001681

Baixeras, E., Huard, B., Miossec, C., Jitsukawa, S., Martin, M., Hercend, T., et al. (1992). Characterization of the Lymphocyte Activation Gene 3-encoded Protein. A New Ligand for Human Leukocyte Antigen Class II Antigens. J. Exp. Med. 176, 327-337. doi:10.1084/jem.176.2.327

Barbari, C., Fontaine, T., Parajuli, P., Lamichhane, N., Jakubski, S., Lamichhane, P., et al. (2020). Immunotherapies and Combination Strategies for ImmunoOncology. Ijms 21, 5009. doi:10.3390/ijms21145009

Baumeister, S. H., Freeman, G. J., Dranoff, G., and Sharpe, A. H. (2016). Coinhibitory Pathways in Immunotherapy for Cancer. Annu. Rev. Immunol. 34, 539-573. doi:10.1146/annurev-immunol-032414-112049

Bendell, J. C., Doi, T., Patel, M. R., Piha-Paul, S. A., Sen, S., Shimizu, T., et al. (2020). A Phase I/II, Two-Part, Multicenter, First-In-Human Study of DS-7300a in Patients with Advanced Solid Malignant Tumors. Jco 38, TPS3646. doi:10.1200/ JCO.2020.38.15_suppl.TPS3646

Bendell, J., Ulahannan, S. V., Chu, Q., Patel, M., George, B., Auguste, A., et al. (2020). Abstract 779: A Phase I Study of BI 754111, an Anti-LAG-3 Monoclonal Antibody (mAb), in Combination with BI 754091, an Anti-PD-1 mAb: Biomarker Analyses from the Microsatellite Stable Metastatic Colorectal Cancer (MSS mCRC) Cohort. Cancer Res. 80, 779. doi:10.1158/1538-7445. Am2020-779

Benzon, B., Zhao, S. G., Haffner, M. C., Takhar, M., Erho, N., Yousefi, K., et al. (2017). Correlation of B7-H3 with Androgen Receptor, Immune Pathways and Poor Outcome in Prostate Cancer: an Expression-Based Analysis. Prostate Cancer Prostatic Dis. 20, 28-35. doi:10.1038/pcan.2016.49

Bever, K. M., Wang, H., Durham, J. N., Petrie, S., Hoare, J., Wilt, C., et al. (2020). Phase II Study of Nivolumab and Relatlimab in Advanced Mismatch Repair Deficient (dMMR) Cancers Resistant to Prior PD-(L)1 Inhibition. Jco 38, TPS839. doi:10.1200/JCO.2020.38.4_suppl.TPS839

Böger, C., Behrens, H.-M., Krüger, S., and Röcken, C. (2017). The Novel Negative Checkpoint Regulator VISTA Is Expressed in Gastric Carcinoma and Associated with PD-L1/pd-1: A Future Perspective for a Combined Gastric Cancer Therapy? Oncoimmunology 6, e1293215. doi:10.1080/2162402X.2017.1293215

Boles, K. S., Vermi, W., Facchetti, F., Fuchs, A., Wilson, T. J., Diacovo, T. G., et al. (2009). A Novel Molecular Interaction for the Adhesion of Follicular CD4 T Cells to Follicular DC. Eur. J. Immunol. 39, 695-703. doi:10.1002/eji. 200839116

Boutros, C., Tarhini, A., Routier, E., Lambotte, O., Ladurie, F. L., Carbonnel, F., et al. (2016). Safety Profiles of Anti-CTLA-4 and Anti-PD-1 Antibodies Alone and in Combination. Nat. Rev. Clin. Oncol. 13, 473-486. doi:10.1038/nrclinonc. 2016.58

Buisson, S., and Triebel, F. (2005). LAG-3 (CD223) Reduces Macrophage and Dendritic Cell Differentiation from Monocyte Precursors. Immunology 114, 369-374. doi:10.1111/j.1365-2567.2004.02087.x

Cai, G., Anumanthan, A., Brown, J. A., Greenfield, E. A., Zhu, B., and Freeman, G. J. (2008). CD160 Inhibits Activation of Human CD4+ T Cells through Interaction with Herpesvirus Entry Mediator. Nat. Immunol. 9, 176-185. doi:10.1038/ ni1554

Cameron, F., Whiteside, G., and Perry, C. (2011). Ipilimumab. Drugs 71, 1093-1104. doi:10.2165/11594010-000000000-00000

Camisaschi, C., Casati, C., Rini, F., Perego, M., De Filippo, A., Triebel, F., et al. (2010). LAG-3 Expression Defines a Subset of CD4+CD25highFoxp3+ Regulatory $\mathrm{T}$ Cells that Are Expanded at Tumor Sites. J. Immunol. 184, 6545-6551. doi:10.4049/jimmunol.0903879

Castellanos, J. R., Purvis, I. J., Labak, C. M., Guda, M. R., Tsung, A. J., Velpula, K. K., et al. (2017). B7-H3 Role in the Immune Landscape of Cancer. Am. J. Clin. Exp. Immunol. 6, 66-75.
Ceeraz, S., Nowak, E. C., and Noelle, R. J. (2013). B7 Family Checkpoint Regulators in Immune Regulation and Disease. Trends Immunology 34, 556-563. doi:10. 1016/j.it.2013.07.003

Chapoval, A. I., Ni, J., Lau, J. S., Wilcox, R. A., Flies, D. B., Liu, D., et al. (2001). B7H3: A Costimulatory Molecule for T Cell Activation and IFN- $\gamma$ Production. Nat. Immunol. 2, 269-274. doi:10.1038/85339

Chauvin, J.-M., Pagliano, O., Fourcade, J., Sun, Z., Wang, H., Sander, C., et al. (2015). TIGIT and PD-1 Impair Tumor Antigen-specific CD8+ $\mathrm{T}$ Cells in Melanoma Patients. J. Clin. Invest. 125, 2046-2058. doi:10. 1172/JCI80445

Chiba, S., Baghdadi, M., Akiba, H., Yoshiyama, H., Kinoshita, I., DosakaAkita, H., et al. (2012). Tumor-infiltrating DCs Suppress Nucleic AcidMediated Innate Immune Responses through Interactions between the Receptor TIM-3 and the Alarmin HMGB1. Nat. Immunol. 13, 832-842. doi:10.1038/ni.2376

Croft, M., So, T., Duan, W., and Soroosh, P. (2009). The Significance of OX40 and OX40L to T-Cell Biology and Immune Disease. Immunological Rev. 229, 173-191. doi:10.1111/j.1600-065X.2009.00766.x

Darvin, P., Toor, S. M., Sasidharan Nair, V., and Elkord, E. (2018). Immune Checkpoint Inhibitors: Recent Progress and Potential Biomarkers. Exp. Mol. Med. 50, 1-11. doi:10.1038/s12276-018-0191-1

Derré, L., Rivals, J.-P., Jandus, C., Pastor, S., Rimoldi, D., Romero, P., et al. (2010). BTLA Mediates Inhibition of Human Tumor-specific CD8+ T Cells that Can Be Partially Reversed by Vaccination. J. Clin. Invest. 120, 157-167. doi:10.1172/ JCI40070

Dirix, L., and Triebel, F. (2019). AIPAC: a Phase IIb Study of Eftilagimod Alpha (IMP321 or LAG-3Ig) Added to Weekly Paclitaxel in Patients with Metastatic Breast Cancer. Future Oncol. 15, 1963-1973. doi:10.2217/fon-2018-0807

Fan, T.-F., Deng, W.-W., Bu, L.-L., Wu, T.-F., Zhang, W.-F., and Sun, Z.-J. (2017). B7-H3 Regulates Migration and Invasion in Salivary Gland Adenoid Cystic Carcinoma via the JAK2/STAT3 Signaling Pathway. Am. J. Transl Res. 9, 1369-1380.

Fan, X., Quezada, S. A., Sepulveda, M. A., Sharma, P., and Allison, J. P. (2014). Engagement of the ICOS Pathway Markedly Enhances Efficacy of CTLA-4 Blockade in Cancer Immunotherapy. J. Exp. Med. 211, 715-725. doi:10.1084/ jem. 20130590

Feeney, K., Kelly, R., Lipton, L. R., Chao, J., Acosta-Rivera, M., Earle, D., et al. (2019). CA224-060: A Randomized, Open Label, Phase II Trial of Relatlimab (Anti-LAG-3) and Nivolumab with Chemotherapy versus Nivolumab with Chemotherapy as First-Line Treatment in Patients with Gastric or Gastroesophageal Junction Adenocarcinoma. Jco 37, TPS4143. doi:10.1200/ JCO.2019.37.15_suppl.TPS4143

Flem-Karlsen, K., Tekle, C., Andersson, Y., Flatmark, K., Fodstad, Ø., and NunesXavier, C. E. (2017). Immunoregulatory Protein B7-H3 Promotes Growth and Decreases Sensitivity to Therapy in Metastatic Melanoma Cells. Pigment Cel Melanoma Res. 30, 467-476. doi:10.1111/pcmr.12599

Flem-Karlsen, K., Tekle, C., Øyjord, T., Flørenes, V. A., Mælandsmo, G. M., Fodstad, Ø., et al. (2019). p38 MAPK Activation through B7-H3-Mediated DUSP10 Repression Promotes Chemoresistance. Sci. Rep. 9, 5839. doi:10.1038/ s41598-019-42303-w

Flies, D. B., Wang, S., Xu, H., and Chen, L. (2011). Cutting Edge: A Monoclonal Antibody Specific for the Programmed Death-1 Homolog Prevents GraftVersus-Host Disease in Mouse Models. J. Immunol. 187, 1537-1541. doi:10. 4049/jimmunol.1100660

Fu, T., He, Q., and Sharma, P. (2011). The ICOS/ICOSL Pathway Is Required for Optimal Antitumor Responses Mediated by Anti-CTLA-4 Therapy. Cancer Res. 71, 5445-5454. doi:10.1158/0008-5472.Can-11-1138

Fuhrman, C. A., Yeh, W.-I., Seay, H. R., Saikumar Lakshmi, P., Chopra, G., Zhang, L., et al. (2015). Divergent Phenotypes of Human Regulatory T Cells Expressing the Receptors TIGIT and CD226. J. Immunol. 195, 145-155. doi:10.4049/ jimmunol.1402381

Gandhi, L., Rodríguez-Abreu, D., Gadgeel, S., Esteban, E., Felip, E., De Angelis, F., et al. (2018). Pembrolizumab Plus Chemotherapy in Metastatic Non-small-cell Lung Cancer. N. Engl. J. Med. 378, 2078-2092. doi:10.1056/NEJMoa1801005

Gao, X., Zhu, Y., Li, G., Huang, H., Zhang, G., Wang, F., et al. (2012). TIM-3 Expression Characterizes Regulatory $\mathrm{T}$ Cells in Tumor Tissues and Is Associated with Lung Cancer Progression. PloS one 7, e30676. doi:10.1371/ journal.pone.0030676 
Gigoux, M., Shang, J., Pak, Y., Xu, M., Choe, J., Mak, T. W., et al. (2009). Inducible Costimulator Promotes Helper T-Cell Differentiation through Phosphoinositide 3-kinase. Proc. Natl. Acad. Sci. 106, 20371-20376. doi:10. 1073/pnas.0911573106

Goding, S. R., Wilson, K. A., Xie, Y., Harris, K. M., Baxi, A., Akpinarli, A., et al. (2013). Restoring Immune Function of Tumor-specific CD4+ T Cells during Recurrence of Melanoma. J. Immunol. 190, 4899-4909. doi:10.4049/jimmunol.1300271

Gu-Trantien, C., Migliori, E., Buisseret, L., de Wind, A., Brohée, S., Garaud, S., et al. (2017). CXCL13-producing TFH Cells Link Immune Suppression and Adaptive Memory in Human Breast Cancer. JCI Insight 2. doi:10.1172/jci. insight. 91487

Guedan, S., Posey, A. D., Jr., Shaw, C., Wing, A., Da, T., Patel, P. R., et al. (2018). Enhancing CAR T Cell Persistence through ICOS and 4-1BB Costimulation. JCI Insight 3, e96976. doi:10.1172/jci.insight.96976

Han, P., Goularte, O. D., Rufner, K., Wilkinson, B., and Kaye, J. (2004). An Inhibitory Ig Superfamily Protein Expressed by Lymphocytes and APCs Is Also an Early Marker of Thymocyte Positive Selection. J. Immunol. 172, 5931-5939. doi:10.4049/jimmunol.172.10.5931

Hanahan, D., and Weinberg, R. A. (2011). Hallmarks of Cancer: the Next Generation. Cell 144, 646-674. doi:10.1016/j.cell.2011.02.013

Harding, J. J., Patnaik, A., Moreno, V., Stein, M., Jankowska, A. M., Velez de Mendizabal, N., et al. (2019). A Phase Ia/Ib Study of an Anti-TIM-3 Antibody (LY3321367) Monotherapy or in Combination with an Anti-PD-L1 Antibody (LY3300054): Interim Safety, Efficacy, and Pharmacokinetic Findings in Advanced Cancers. Jco 37, 12. doi:10.1200/JCO.2019.37.8_suppl.12

Hellmann, M. D., Ciuleanu, T.-E., Pluzanski, A., Lee, J. S., Otterson, G. A., Audigier-Valette, C., et al. (2018). Nivolumab Plus Ipilimumab in Lung Cancer with a High Tumor Mutational Burden. N. Engl. J. Med. 378, 2093-2104. doi:10.1056/NEJMoa1801946

Huang, C.-T., Workman, C. J., Flies, D., Pan, X., Marson, A. L., Zhou, G., et al. (2004). Role of LAG-3 in Regulatory T Cells. Immunity 21, 503-513. doi:10. 1016/j.immuni.2004.08.010

Huang, R.-Y., Eppolito, C., Lele, S., Shrikant, P., Matsuzaki, J., and Odunsi, K. (2015). LAG3 and PD1 Co-inhibitory Molecules Collaborate to Limit CD8+ $\mathrm{T}$ Cell Signaling and Dampen Antitumor Immunity in a Murine Ovarian Cancer Model. Oncotarget 6, 27359-27377. doi:10.18632/oncotarget.4751

Huang, X., Bai, X., Cao, Y., Wu, J., Huang, M., Tang, D., et al. (2010). Lymphoma Endothelium Preferentially Expresses Tim- 3 and Facilitates the Progression of Lymphoma by Mediating Immune Evasion. J. Exp. Med. 207, 505-520. doi:10. 1084/jem.20090397

Huang, Y.-H., Zhu, C., Kondo, Y., Anderson, A. C., Gandhi, A., Russell, A., et al. (2015). CEACAM1 Regulates TIM-3-Mediated Tolerance and Exhaustion. Nature 517, 386-390. doi:10.1038/nature13848

Huard, B., Mastrangeli, R., Prigent, P., Bruniquel, D., Donini, S., El-Tayar, N., et al. (1997). Characterization of the Major Histocompatibility Complex Class II Binding Site on LAG-3 Protein. Proc. Natl. Acad. Sci. 94, 5744-5749. doi:10. 1073/pnas.94.11.5744

Huard, B., Prigent, P., Tournier, M., Bruniquel, D., and Triebel, F. (1995). CD4/ major Histocompatibility Complex Class II Interaction Analyzed with CD4and Lymphocyte Activation Gene-3 (LAG-3)-Ig Fusion Proteins. Eur. J. Immunol. 25, 2718-2721. doi:10.1002/eji.1830250949

Huard, B., Tournier, M., Hercend, T., Triebel, F., and Faure, F. (1994). Lymphocyte-activation Gene 3/major Histocompatibility Complex Class II Interaction Modulates the Antigenic Response of CD4+ T Lymphocytes. Eur. J. Immunol. 24, 3216-3221. doi:10.1002/eji.1830241246

Hutloff, A., Dittrich, A. M., Beier, K. C., Eljaschewitsch, B., Kraft, R., Anagnostopoulos, I., et al. (1999). ICOS Is an Inducible T-Cell Costimulator Structurally and Functionally Related to CD28. Nature 397, 263-266. doi:10.1038/16717

Jan, M., Chao, M. P., Cha, A. C., Alizadeh, A. A., Gentles, A. J., Weissman, I. L., et al. (2011). Prospective Separation of Normal and Leukemic Stem Cells Based on Differential Expression of TIM3, a Human Acute Myeloid Leukemia Stem Cell Marker. Proc. Natl. Acad. Sci. 108, 5009-5014. doi:10.1073/pnas. 1100551108

Janakiram, M., Shah, U. A., Liu, W., Zhao, A., Schoenberg, M. P., and Zang, X. (2017). The Third Group of the B7-CD28 Immune Checkpoint Family: HHLA2, TMIGD2, B7x, and B7-H3. Immunol. Rev. 276, 26-39. doi:10. 1111/imr.12521
Jiang, B., Liu, F., Liu, Z., Zhang, T., and Hua, D. (2016). B7-H3 Increases Thymidylate Synthase Expression via the PI3k-Akt Pathway. Tumor Biol. 37, 9465-9472. doi:10.1007/s13277-015-4740-0

Jin, Y., Zhang, P., Li, J., Zhao, J., Liu, C., Yang, F., et al. (2015). B7-H3 in Combination with Regulatory T Cell Is Associated with Tumor Progression in Primary Human Non-small Cell Lung Cancer. Int. J. Clin. Exp. Pathol. 8, 13987-13995.

Johnson, M. L., Patel, M. R., Cherry, M., Kang, Y.-K., Yamaguchi, K., Oh, D.-Y., et al. (2020). Safety of BI 754111, an Anti-LAG-3 Monoclonal Antibody (mAb), in Combination with BI 754091, an Anti-PD-1 mAb, in Patients with Advanced Solid Tumors. Jco 38, 3063. doi:10.1200/JCO.2020.38.15_suppl.3063

Johnston, R. J., Comps-Agrar, L., Hackney, J., Yu, X., Huseni, M., Yang, Y., et al. (2014). The Immunoreceptor TIGIT Regulates Antitumor and Antiviral CD8 + T Cell Effector Function. Cancer cell 26, 923-937. doi:10.1016/j.ccell.2014.10.018

Joller, N., Lozano, E., Burkett, P. R., Patel, B., Xiao, S., Zhu, C., et al. (2014). Treg Cells Expressing the Coinhibitory Molecule TIGIT Selectively Inhibit Proinflammatory Th1 and Th17 Cell Responses. Immunity 40, 569-581. doi:10.1016/j.immuni.2014.02.012

Kang, C.-W., Dutta, A., Chang, L.-Y., Mahalingam, J., Lin, Y.-C., Chiang, J.-M., et al. (2015). Apoptosis of Tumor Infiltrating Effector TIM-3+CD8+ T Cells in Colon Cancer. Sci. Rep. 5, 15659. doi:10.1038/srep15659

Kang, F.-b., Wang, L., Jia, H.-c., Li, D., Li, H.-j., Zhang, Y.-g., et al. (2015). B7-H3 Promotes Aggression and Invasion of Hepatocellular Carcinoma by Targeting Epithelial-To-Mesenchymal Transition via JAK2/STAT3/Slug Signaling Pathway. Cancer Cel Int. 15, 45. doi:10.1186/s12935-015-0195-z

Khayyamian, S., Hutloff, A., Büchner, K., Gräfe, M., Henn, V., Kroczek, R. A., et al. (2002). ICOS-ligand, Expressed on Human Endothelial Cells, Costimulates Th1 and Th2 Cytokine Secretion by Memory CD4+ T Cells. Proc. Natl. Acad. Sci. 99, 6198-6203. doi:10.1073/pnas.092576699

Kisielow, M., Kisielow, J., Capoferri-Sollami, G., and Karjalainen, K. (2005). Expression of Lymphocyte Activation Gene 3 (LAG-3) on B Cells Is Induced by T Cells. Eur. J. Immunol. 35, 2081-2088. doi:10.1002/eji.200526090

Kondo, Y., Ohno, T., Nishii, N., Harada, K., Yagita, H., and Azuma, M. (2016). Differential Contribution of Three Immune Checkpoint (VISTA, CTLA-4, PD1) Pathways to Antitumor Responses against Squamous Cell Carcinoma. Oral Oncol. 57, 54-60. doi:10.1016/j.oraloncology.2016.04.005

Kouo, T., Huang, L., Pucsek, A. B., Cao, M., Solt, S., Armstrong, T., et al. (2015). Galectin-3 Shapes Antitumor Immune Responses by Suppressing CD8+ T Cells via LAG-3 and Inhibiting Expansion of Plasmacytoid Dendritic Cells. Cancer Immunol. Res. 3, 412-423. doi:10.1158/2326-6066.Cir-14-0150

Kourepini, E., Paschalidis, N., Simoes, D. C., Aggelakopoulou, M., Grogan, J. L., and Panoutsakopoulou, V. (2016). TIGIT Enhances Antigen-specific Th2 Recall Responses and Allergic Disease. J. Immunol. 196, 3570-3580. doi:10. 4049/jimmunol.1501591

Koyama, S., Akbay, E. A., Li, Y. Y., Herter-Sprie, G. S., Buczkowski, K. A., Richards, W. G., et al. (2016). Adaptive Resistance to Therapeutic PD-1 Blockade Is Associated with Upregulation of Alternative Immune Checkpoints. Nat. Commun. 7, 10501. doi:10.1038/ncomms10501

Kuklinski, L. F., Yan, S., Li, Z., Fisher, J. L., Cheng, C., Noelle, R. J., et al. (2018). VISTA Expression on Tumor-Infiltrating Inflammatory Cells in Primary Cutaneous Melanoma Correlates with Poor Disease-specific Survival. Cancer Immunol. Immunother. 67, 1113-1121. doi:10.1007/s00262-018-2169-1

Kurtulus, S., Sakuishi, K., Ngiow, S.-F., Joller, N., Tan, D. J., Teng, M. W. L., et al. (2015). TIGIT Predominantly Regulates the Immune Response via Regulatory T Cells. J. Clin. Invest. 125, 4053-4062. doi:10.1172/jci81187

Lakhani, N., Spreafico, A., Tolcher, A. W., Rodon, J., Janku, F., Chandana, S. R., et al. (2020). 10190 Phase I Studies of Sym021, an Anti-PD-1 Antibody, Alone and in Combination with Sym022 (Anti-LAG-3) or Sym023 (Anti-TIM-3). Ann. Oncol. 31, S704. doi:10.1016/j.annonc.2020.08.1139

Le Mercier, I., Chen, W., Lines, J. L., Day, M., Li, J., Sergent, P., et al. (2014). VISTA Regulates the Development of Protective Antitumor Immunity. Cancer Res. 74, 1933-1944. doi:10.1158/0008-5472.CAN-13-1506

Levin, S. D., Taft, D. W., Brandt, C. S., Bucher, C., Howard, E. D., Chadwick, E. M., et al. (2011). Vstm3 Is a Member of the CD28 Family and an Important Modulator of T-Cell Function. Eur. J. Immunol. 41, 902-915. doi:10.1002/eji. 201041136

Li, F.-J., Zhang, Y., Jin, G.-X., Yao, L., and Wu, D.-Q. (2013). Expression of LAG-3 Is Coincident with the Impaired Effector Function of HBV-specific CD8+ 
T Cell in HCC Patients. Immunol. Lett. 150, 116-122. doi:10.1016/j.imlet.2012. 12.004

Li, M., Xia, P., Du, Y., Liu, S., Huang, G., Chen, J., et al. (2014). T-cell Immunoglobulin and ITIM Domain (TIGIT) Receptor/Poliovirus Receptor (PVR) Ligand Engagement Suppresses Interferon- $\gamma$ Production of Natural Killer Cells via $\beta$-Arrestin 2-mediated Negative Signaling. J. Biol. Chem. 289, 17647-17657. doi:10.1074/jbc.M114.572420

Li, M., Zhang, G., Zhang, X., Lv, G., Wei, X., Yuan, H., et al. (2014). Overexpression of B7-H3 in CD14+ Monocytes Is Associated with Renal Cell Carcinoma Progression. Med. Oncol. 31, 349. doi:10.1007/s12032-014-0349-1

Li, N., Wang, Y., Forbes, K., Vignali, K. M., Heale, B. S., Saftig, P., et al. (2007). Metalloproteases Regulate T-Cell Proliferation and Effector Function via LAG-

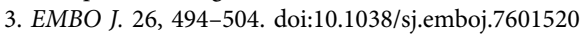

Lines, J. L., Sempere, L. F., Broughton, T., Wang, L., and Noelle, R. (2014). VISTA Is a Novel Broad-Spectrum Negative Checkpoint Regulator for Cancer Immunotherapy. Cancer Immunol. Res. 2, 510-517. doi:10.1158/2326-6066. Cir-14-0072

Lipson, E. J., Long, G. V., Tawbi, H., Schadendorf, D., Atkinson, V. G., Maurer, M., et al. (2018). CA224-047: A Randomized, Double-Blind, Phase II/III Study of Relatlimab (Anti-LAG-3) in Combination with Nivolumab (AntiPD-1) versus Nivolumab Alone in Previously Untreated Metastatic or Unresectable Melanoma. Ann. Oncol. 29, viii464-viii465. doi:10.1093/ annonc/mdy 289.058

Liu, H., Tekle, C., Chen, Y.-W., Kristian, A., Zhao, Y., Zhou, M., et al. (2011). B7H3 Silencing Increases Paclitaxel Sensitivity by Abrogating Jak2/Stat3 Phosphorylation. Mol. Cancer Ther. 10, 960-971. doi:10.1158/1535-7163. Mct-11-0072

Liu, J., Yuan, Y., Chen, W., Putra, J., Suriawinata, A. A., Schenk, A. D., et al. (2015). Immune-checkpoint Proteins VISTA and PD-1 Nonredundantly Regulate Murine T-Cell Responses. Proc. Natl. Acad. Sci. USA 112, 6682-6687. doi:10.1073/pnas.1420370112

Llosa, N. J., Cruise, M., Tam, A., Wicks, E. C., Hechenbleikner, E. M., Taube, J. M., et al. (2015). The Vigorous Immune Microenvironment of Microsatellite Instable Colon Cancer Is Balanced by Multiple Counter-inhibitory Checkpoints. Cancer Discov. 5, 43-51. doi:10.1158/2159-8290.Cd-14-0863

Loeser, H., Kraemer, M., Gebauer, F., Bruns, C., Schröder, W., Zander, T., et al. (2019). The Expression of the Immune Checkpoint Regulator VISTA Correlates with Improved Overall Survival in pT1/2 Tumor Stages in Esophageal Adenocarcinoma. Oncoimmunology 8, e1581546. doi:10.1080/2162402x.2019. 1581546

Lozano, E., Dominguez-Villar, M., Kuchroo, V., and Hafler, D. A. (2012). The TIGIT/CD226 axis Regulates Human T Cell Function. J. Immunol. 188, 3869-3875. doi:10.4049/jimmunol.1103627

Luke, J. J., Patel, M. R., Hamilton, E. P., Chmielowski, B., Ulahannan, S. V., Kindler, H. L., et al. (2020). A Phase I, First-In-Human, Open-Label, Dose-Escalation Study of MGD013, a Bispecific DART Molecule Binding PD-1 and LAG-3, in Patients with Unresectable or Metastatic Neoplasms. Jco 38, 3004. doi:10.1200/ JCO.2020.38.15_suppl.3004

Manieri, N. A., Chiang, E. Y., and Grogan, J. L. (2017). TIGIT: A Key Inhibitor of the Cancer Immunity Cycle. Trends Immunology 38, 20-28. doi:10.1016/j.it. 2016.10.002

Mao, X., Ou, M. T., Karuppagounder, S. S., Kam, T.-I., Yin, X., Xiong, Y., et al. (2016). Pathological -synuclein Transmission Initiated by Binding LymphocyteActivation Gene 3. Science 353, aah3374. doi:10.1126/science.aah3374

Mao, Y., Chen, L., Wang, F., Zhu, D., Ge, X., Hua, D., et al. (2017). Cancer Cellexpressed B7H3 Regulates the Differentiation of Tumorassociated Macrophages in Human Colorectal Carcinoma. Oncol. Lett. 14, 6177-6183. doi:10.3892/ol.2017.6935

Marabelle, A., Fakih, M., Lopez, J., Shah, M., Shapira-Frommer, R., Nakagawa, K., et al. (2020). Association of Tumour Mutational Burden with Outcomes in Patients with Advanced Solid Tumours Treated with Pembrolizumab: Prospective Biomarker Analysis of the Multicohort, Open-Label, Phase 2 KEYNOTE-158 Study. Lancet Oncol. 21, 1353-1365. doi:10.1016/s14702045(20)30445-9

Marcus, L., Lemery, S. J., Keegan, P., and Pazdur, R. (2019). FDA Approval Summary: Pembrolizumab for the Treatment of Microsatellite Instability-High Solid Tumors. Clin. Cancer Res. 25, 3753-3758. doi:10.1158/1078-0432.Ccr-184070
Marinelli, O., Nabissi, M., Morelli, M. B., Torquati, L., Amantini, C., and Santoni, G. (2018). ICOS-L as a Potential Therapeutic Target for Cancer Immunotherapy. Cpps 19, 1107-1113. doi:10.2174/1389203719666180608093913

Martin-Orozco, N., Li, Y., Wang, Y., Liu, S., Hwu, P., Liu, Y.-J., et al. (2010). Melanoma Cells Express ICOS Ligand to Promote the Activation and Expansion of T-Regulatory Cells. Cancer Res. 70, 9581-9590. doi:10.1158/ 0008-5472.Can-10-1379

Matsuzaki, J., Gnjatic, S., Mhawech-Fauceglia, P., Beck, A., Miller, A., Tsuji, T., et al. (2010). Tumor-infiltrating NY-ESO-1-specific CD8+T Cells Are Negatively Regulated by LAG-3 and PD-1 in Human Ovarian Cancer. Proc. Natl. Acad. Sci. USA 107, 7875-7880. doi:10.1073/pnas.1003345107

Modak, S., Carrasquillo, J., LaQuaglia, M., Pat, Z., Heaton, T., Cheung, N.-K., et al. (2018). Abstract CT006: Intraperitoneal Radioimmunotherapy for Desmoplastic Small Round Cell Tumor: Results of a Phase I Study (NCT01099644). Cancer Res. 78, CT006. doi:10.1158/1538-7445.Am2018-ct006

Monney, L., Sabatos, C. A., Gaglia, J. L., Ryu, A., Waldner, H., Chernova, T., et al. (2002). Th1-specific Cell Surface Protein Tim-3 Regulates Macrophage Activation and Severity of an Autoimmune Disease. Nature 415, 536-541. doi:10.1038/415536a

Motzer, R. J., Tannir, N. M., McDermott, D. F., Arén Frontera, O., Melichar, B., Choueiri, T. K., et al. (2018). Nivolumab Plus Ipilimumab versus Sunitinib in Advanced Renal-Cell Carcinoma. N. Engl. J. Med. 378, 1277-1290. doi:10.1056/ NEJMoa1712126

Murphy, K. M., Nelson, C. A., and Šedý, J. R. (2006). Balancing Co-stimulation and Inhibition with BTLA and HVEM. Nat. Rev. Immunol. 6, 671-681. doi:10.1038/ nri1917

Musielak, B., Kocik, J., Skalniak, L., Magiera-Mularz, K., Sala, D., Czub, M., et al. (2019). CA-170 - a Potent Small-Molecule PD-L1 Inhibitor or Not? Molecules 24 (15), 2804. doi:10.3390/molecules24152804

Nagahara, K., Arikawa, T., Oomizu, S., Kontani, K., Nobumoto, A., Tateno, H., et al. (2008). Galectin-9 Increases Tim-3+ Dendritic Cells and CD8+ T Cells and Enhances Antitumor Immunity via Galectin-9-Tim-3 Interactions. J. Immunol. 181, 7660-7669. doi:10.4049/jimmunol.181.11.7660

Nagase, H., Takeoka, T., Urakawa, S., Morimoto-Okazawa, A., Kawashima, A., Iwahori, K., et al. (2017). ICOS+Foxp3+TILs in Gastric Cancer Are Prognostic Markers and Effector Regulatory T Cells Associated withHelicobacter Pylori. Int. J. Cancer 140, 686-695. doi:10.1002/ijc.30475

Nakayama, M., Akiba, H., Takeda, K., Kojima, Y., Hashiguchi, M., Azuma, M., et al. (2009). Tim-3 Mediates Phagocytosis of Apoptotic Cells and CrossPresentation. Blood 113, 3821-3830. doi:10.1182/blood-2008-10-185884

Ngiow, S. F., von Scheidt, B., Akiba, H., Yagita, H., Teng, M. W. L., and Smyth, M. J. (2011). Anti-TIM3 Antibody Promotes T Cell IFN- $\gamma$-Mediated Antitumor Immunity and Suppresses Established Tumors. Cancer Res. 71, 3540-3551. doi:10.1158/0008-5472.Can-11-0096

Niu, J., Nagrial, A., Voskoboynik, M., Chung, H. C., Lee, D. H., Ahn, M.-J., et al. (2020). 1410P Safety and Efficacy of Vibostolimab, an Anti-TIGIT Antibody, Plus Pembrolizumab in Patients with Anti-PD-1/pd-L1-naive NSCLC. Ann. Oncol. 31, S891-S892. doi:10.1016/j.annonc.2020.08.1724

Papadopoulos, K. P., Lakhani, N. J., Johnson, M. L., Park, H., Wang, D., Yap, T. A., et al. (2019). First-in-human Study of REGN3767 (R3767), a Human LAG-3 Monoclonal Antibody (mAb), \pm Cemiplimab in Patients (Pts) with Advanced Malignancies. Jco 37, 2508. doi:10.1200/JCO.2019.37.15_suppl.2508

Pardoll, D. M. (2012). The Blockade of Immune Checkpoints in Cancer Immunotherapy. Nat. Rev. Cancer 12, 252-264. doi:10.1038/nrc3239

Paulos, C. M., and June, C. H. (2010). Putting the Brakes on BTLA in T CellMediated Cancer Immunotherapy. J. Clin. Invest. 120, 76-80. doi:10.1172/ jci41811

Peguero, J. A., Bajaj, P., Carcereny, E., Clay, T. D., Doger, B., Felip, E., et al. (2019). A Multicenter, Phase II Study of Soluble LAG-3 (Eftilagimod Alpha) in Combination with Pembrolizumab (TACTI-002) in Patients with Advanced Non-small Cell Lung Cancer (NSCLC) or Head and Neck Squamous Cell Carcinoma (HNSCC). Jco 37, TPS2667. doi:10.1200/JCO.2019.37.15_suppl. TPS2667

Popovic, A., Jaffee, E. M., and Zaidi, N. (2018). Emerging Strategies for Combination Checkpoint Modulators in Cancer Immunotherapy. J. Clin. Invest. 128, 3209-3218. doi:10.1172/jci120775

Powderly, J., Cote, G., Flaherty, K., Szmulewitz, R. Z., Ribas, A., Weber, J., et al. (2015). Interim Results of an Ongoing Phase I, Dose Escalation Study of 
MGA271 (Fc-Optimized Humanized Anti-B7-H3 Monoclonal Antibody) in Patients with Refractory B7-H3-Expressing Neoplasms or Neoplasms Whose Vasculature Expresses B7-H3. J. Immunother. Cancer 3, O8. doi:10.1186/20511426-3-S2-O8

Powderly, J., Patel, M. R., Lee, J. J., Brody, J., Meric-Bernstam, F., Hamilton, E., et al. (2017). CA-170, a First in Class Oral Small Molecule Dual Inhibitor of Immune Checkpoints PD-L1 and VISTA, Demonstrates Tumor Growth Inhibition in Pre-clinical Models and Promotes T Cell Activation in Phase 1 Study. Ann. Oncol. 28, v405-v406. doi:10.1093/annonc/mdx376.007

Prasad, D. V. R., Nguyen, T., Li, Z., Yang, Y., Duong, J., Wang, Y., et al. (2004). Murine B7-H3 Is a Negative Regulator of T Cells. J. Immunol. 173, 2500-2506. doi:10.4049/jimmunol.173.4.2500

Qin, S., Xu, L., Yi, M., Yu, S., Wu, K., and Luo, S. (2019). Novel Immune Checkpoint Targets: Moving beyond PD-1 and CTLA-4. Mol. Cancer 18, 155. doi:10.1186/s12943-019-1091-2

Rini, B. I., Plimack, E. R., Stus, V., Gafanov, R., Hawkins, R., Nosov, D., et al. (2019). Pembrolizumab Plus Axitinib versus Sunitinib for Advanced RenalCell Carcinoma. N. Engl. J. Med. 380, 1116-1127. doi:10.1056/ NEJMoa 1816714

Rizvi, N. A., Hellmann, M. D., Brahmer, J. R., Juergens, R. A., Borghaei, H., Gettinger, S., et al. (2016). Nivolumab in Combination with Platinum-Based Doublet Chemotherapy for First-Line Treatment of Advanced Non-small-cell Lung Cancer. Jco 34, 2969-2979. doi:10.1200/jco.2016.66.9861

Rodriguez-Abreu, D., Johnson, M. L., Hussein, M. A., Cobo, M., Patel, A. J., Secen, N. M., et al. (2020). Primary Analysis of a Randomized, Double-Blind, Phase II Study of the Anti-TIGIT Antibody Tiragolumab (Tira) Plus Atezolizumab (Atezo) versus Placebo Plus Atezo as First-Line (1L) Treatment in Patients with PD-L1-Selected NSCLC (CITYSCAPE). Jco 38, 9503. doi:10.1200/JCO.2020.38. 15_suppl.9503

Sakuishi, K., Apetoh, L., Sullivan, J. M., Blazar, B. R., Kuchroo, V. K., and Anderson, A. C. (2010). Targeting Tim-3 and PD-1 Pathways to Reverse T Cell Exhaustion and Restore Anti-tumor Immunity. J. Exp. Med. 207, 2187-2194. doi:10.1084/jem.20100643

Sedy, J. R., Gavrieli, M., Potter, K. G., Hurchla, M. A., Lindsley, R. C., Hildner, K., et al. (2005). B and T Lymphocyte Attenuator Regulates T Cell Activation through Interaction with Herpesvirus Entry Mediator. Nat. Immunol. 6, 90-98. doi:10.1038/ni1144

Sharma, P., Hu-Lieskovan, S., Wargo, J. A., and Ribas, A. (2017). Primary, Adaptive, and Acquired Resistance to Cancer Immunotherapy. Cell 168, 707-723. doi:10.1016/j.cell.2017.01.017

Solinas, C., Gu-Trantien, C., and Willard-Gallo, K. (2020). The Rationale behind Targeting the ICOS-ICOS Ligand Costimulatory Pathway in Cancer Immunotherapy. ESMO Open 5, e000544. doi:10.1136/esmoopen-2019-000544

Steinberg, M. W., Cheung, T. C., and Ware, C. F. (2011). The Signaling Networks of the Herpesvirus Entry Mediator (TNFRSF14) in Immune Regulation. Immunological Rev. 244, 169-187. doi:10.1111/j.1600-065X. 2011.01064.x

Stengel, K. F., Harden-Bowles, K., Yu, X., Rouge, L., Yin, J., Comps-Agrar, L., et al. (2012). Structure of TIGIT Immunoreceptor Bound to Poliovirus Receptor Reveals a Cell-Cell Adhesion and Signaling Mechanism that Requires Cis-Trans Receptor Clustering. Proc. Natl. Acad. Sci. 109, 5399-5404. doi:10.1073/pnas. 1120606109

Strauss, L., Bergmann, C., Szczepanski, M. J., Lang, S., Kirkwood, J. M., and Whiteside, T. L. (2008). Expression of ICOS on Human Melanoma-Infiltrating CD4+CD25highFoxp3+ T Regulatory Cells: Implications and Impact on Tumor-Mediated Immune Suppression. J. Immunol. 180, 2967-2980. doi:10. 4049/jimmunol.180.5.2967

Suh, W.-K., Gajewska, B. U., Okada, H., Gronski, M. A., Bertram, E. M., Dawicki, W., et al. (2003). The B7 Family Member B7-H3 Preferentially Down-Regulates T Helper Type 1-mediated Immune Responses. Nat. Immunol. 4, 899-906. doi:10.1038/ni967

Swallow, M. M., Wallin, J. J., and Sha, W. C. (1999). B7h, a Novel Costimulatory Homolog of B7.1 and B7.2, Is Induced by TNFa. Immunity 11, 423-432. doi:10. 1016/s1074-7613(00)80117-x

Taube, J. M., Young, G. D., McMiller, T. L., Chen, S., Salas, J. T., Pritchard, T. S., et al. (2015). Differential Expression of Immune-Regulatory Genes Associated with PD-L1 Display in Melanoma: Implications for PD-1 Pathway Blockade. Clin. Cancer Res. 21, 3969-3976. doi:10.1158/1078-0432.Ccr-15-0244
Torphy, R., Schulick, R., and Zhu, Y. (2017). Newly Emerging Immune Checkpoints: Promises for Future Cancer Therapy. Ijms 18, 2642. doi:10. 3390/ijms18122642

Trebeschi, S., Drago, S. G., Birkbak, N. J., Kurilova, I., Călin, A. M., Delli Pizzi, A., et al. (2019). Predicting Response to Cancer Immunotherapy Using Noninvasive Radiomic Biomarkers. Ann. Oncol. 30, 998-1004. doi:10.1093/ annonc/mdz108

Triebel, F., Hacene, K., and Pichon, M.-F. (2006). A Soluble Lymphocyte Activation Gene-3 (sLAG-3) Protein as a Prognostic Factor in Human Breast Cancer Expressing Estrogen or Progesterone Receptors. Cancer Lett. 235, 147-153. doi:10.1016/j.canlet.2005.04.015

Triebel, F., Jitsukawa, S., Baixeras, E., Roman-Roman, S., Genevee, C., ViegasPequignot, E., et al. (1990). LAG-3, a Novel Lymphocyte Activation Gene Closely Related to CD4. J. Exp. Med. 171, 1393-1405. doi:10.1084/jem.171.5. 1393

Uboha, N. V., Milhem, M. M., Kovacs, C., Amin, A., Magley, A., Purkayastha, D. D., et al. (2019). Phase II Study of Spartalizumab (PDR001) and LAG525 in Advanced Solid Tumors and Hematologic Malignancies. Jco 37, 2553. doi:10. 1200/JCO.2019.37.15_suppl.2553

Vaddepally, R. K., Kharel, P., Pandey, R., Garje, R., and Chandra, A. B. (2020). Review of Indications of FDA-Approved Immune Checkpoint Inhibitors Per NCCN Guidelines with the Level of Evidence. Cancers 12, 738. doi:10.3390/ cancers 12030738

van de Weyer, P. S., Muehlfeit, M., Klose, C., Bonventre, J. V., Walz, G., and Kuehn, E. W. (2006). A Highly Conserved Tyrosine of Tim-3 Is Phosphorylated upon Stimulation by its Ligand Galectin-9. Biochem. Biophysical Res. Commun. 351, 571-576. doi:10.1016/j.bbrc.2006.10.079

Vinay, D. S., Ryan, E. P., Pawelec, G., Talib, W. H., Stagg, J., Elkord, E., et al. (2015). Immune Evasion in Cancer: Mechanistic Basis and Therapeutic Strategies. Semin. Cancer Biol. 35, S185-S198. doi:10.1016/j.semcancer.2015.03.004

Wang, D. Y., Salem, J.-E., Cohen, J. V., Chandra, S., Menzer, C., Ye, F., et al. (2018). Fatal Toxic Effects Associated with Immune Checkpoint Inhibitors. JAMA Oncol. 4, 1721-1728. doi:10.1001/jamaoncol.2018.3923

Wang, J., Sanmamed, M. F., Datar, I., Su, T. T., Ji, L., Sun, J., et al. (2019). Fibrinogen-like Protein 1 Is a Major Immune Inhibitory Ligand of LAG-3. Cell 176, 334-347. doi:10.1016/j.cell.2018.11.010

Wang, J., Wu, G., Manick, B., Hernandez, V., Renelt, M., Erickson, C., et al. (2019). VSIG-3 as a Ligand of VISTA Inhibits Human T-Cell Function. Immunology 156, 74-85. doi:10.1111/imm.13001

Wang, L., Rubinstein, R., Lines, J. L., Wasiuk, A., Ahonen, C., Guo, Y., et al. (2011). VISTA, a Novel Mouse Ig Superfamily Ligand that Negatively Regulates T Cell Responses. J. Exp. Med. 208, 577-592. doi:10.1084/jem.20100619

Wang, Y., Deng, W., Li, N., Neri, S., Sharma, A., Jiang, W., et al. (2018). Combining Immunotherapy and Radiotherapy for Cancer Treatment: Current Challenges and Future Directions. Front. Pharmacol. 9, 185. doi:10.3389/fphar.2018.00185

Woo, S.-R., Turnis, M. E., Goldberg, M. V., Bankoti, J., Selby, M., Nirschl, C. J., et al. (2012). Immune Inhibitory Molecules LAG-3 and PD-1 Synergistically Regulate T-Cell Function to Promote Tumoral Immune Escape. Cancer Res. 72, 917-927. doi:10.1158/0008-5472.Can-11-1620

Wu, L., Deng, W.-W., Huang, C.-F., Bu, L.-L., Yu, G.-T., Mao, L., et al. (2017). Expression of VISTA Correlated with Immunosuppression and Synergized with CD8 to Predict Survival in Human Oral Squamous Cell Carcinoma. Cancer Immunol. Immunother. 66, 627-636. doi:10.1007/s00262-0171968-0

Xie, C., Liu, D., Chen, Q., Yang, C., Wang, B., and Wu, H. (2016). Soluble B7-H3 Promotes the Invasion and Metastasis of Pancreatic Carcinoma Cells through the TLR4/NF-Kb Pathway. Sci. Rep. 6, 27528. doi:10.1038/srep27528

Xin Yu, J., Hubbard-Lucey, V. M., and Tang, J. (2019). Immuno-oncology Drug Development Goes Global. Nat. Rev. Drug Discov. 18, 899-900. doi:10.1038/ d41573-019-00167-9

Yang, S., Wei, W., and Zhao, Q. (2020). B7-H3, a Checkpoint Molecule, as a Target for Cancer Immunotherapy. Int. J. Biol. Sci. 16, 1767-1773. doi:10.7150/ijbs. 41105

Yap, T. A., Burris, H. A., Kummar, S., Falchook, G. S., Pachynski, R. K., LoRusso, P., et al. (2018). ICONIC: Biologic and Clinical Activity of First in Class ICOS Agonist Antibody JTX-2011 +/- Nivolumab (Nivo) in Patients (Pts) with Advanced Cancers. Jco 36, 3000. doi:10.1200/JCO.2018.36.15_suppl.3000 
Yoon, K. W., Byun, S., Kwon, E., Hwang, S.-Y., Chu, K., Hiraki, M., et al. (2015). Control of Signaling-Mediated Clearance of Apoptotic Cells by the Tumor Suppressor P53. Science 349, 1261669. doi:10.1126/science.1261669

Yoshinaga, S. K., Whoriskey, J. S., Khare, S. D., Sarmiento, U., Guo, J., Horan, T., et al. (1999). T-cell Co-stimulation through B7RP-1 and ICOS. Nature 402, 827-832. doi:10.1038/45582

Yu, X., Harden, K., C Gonzalez, L., Francesco, M., Chiang, E., Irving, B., et al. (2009). The Surface Protein TIGIT Suppresses T Cell Activation by Promoting the Generation of Mature Immunoregulatory Dendritic Cells. Nat. Immunol. 10, 48-57. doi:10.1038/ni.1674

Zhang, J., Liu, L., Han, S., Li, Y., Qian, Q., Zhang, Q., et al. (2017). B7-H3 Is Related to Tumor Progression in Ovarian Cancer. Oncol. Rep. 38, 2426-2434. doi:10. 3892/or.2017.5858

Zhang, M., Pang, H.-J., Zhao, W., Li, Y.-F., Yan, L.-X., Dong, Z.-Y., et al. (2018). VISTA Expression Associated with CD8 Confers a Favorable Immune Microenvironment and Better Overall Survival in Hepatocellular Carcinoma. BMC Cancer 18, 511. doi:10.1186/s12885-018-4435-1

Zhang, P., Yu, S., Li, H., Liu, C., Li, J., Lin, W., et al. (2015). ILT4 Drives B7-H3 Expression via PI3K/AKT/mTOR Signalling and ILT4/B7-H3 Co-expression Correlates with Poor Prognosis in Non-small Cell Lung Cancer. FEBS Lett. 589, 2248-2256. doi:10.1016/j.febslet.2015.06.037

Zhang, Q., Bi, J., Zheng, X., Chen, Y., Wang, H., Wu, W., et al. (2018). Blockade of the Checkpoint Receptor TIGIT Prevents NK Cell Exhaustion and Elicits Potent Anti-tumor Immunity. Nat. Immunol. 19, 723-732. doi:10.1038/ s41590-018-0132-0
Zhang, Y., Cai, P., Liang, T., Wang, L., and Hu, L. (2017). TIM-3 Is a Potential Prognostic Marker for Patients with Solid Tumors: A Systematic Review and Meta-Analysis. Oncotarget 8, 31705-31713. doi:10.18632/oncotarget. 15954

Zhang, Y., Luo, Y., Qin, S.-L., Mu, Y.-F., Qi, Y., Yu, M.-H., et al. (2016). The Clinical Impact of ICOS Signal in Colorectal Cancer Patients. Oncoimmunology 5, e1141857. doi:10.1080/2162402X.2016.1141857

Zhu, C., Anderson, A. C., Schubart, A., Xiong, H., Imitola, J., Khoury, S. J., et al. (2005). The Tim-3 Ligand Galectin-9 Negatively Regulates T Helper Type 1 Immunity. Nat. Immunol. 6, 1245-1252. doi:10.1038/ni1271

Zong, L., Zhou, Y., Zhang, M., Chen, J., and Xiang, Y. (2020). VISTA Expression Is Associated with a Favorable Prognosis in Patients with High-Grade Serous Ovarian Cancer. Cancer Immunol. Immunother. 69, 33-42. doi:10.1007/ s00262-019-02434-5

Conflict of Interest: The authors declare that the research was conducted in the absence of any commercial or financial relationships that could be construed as a potential conflict of interest.

Copyright () 2021 Lee, Ha and Kim. This is an open-access article distributed under the terms of the Creative Commons Attribution License (CC BY). The use, distribution or reproduction in other forums is permitted, provided the original author(s) and the copyright owner(s) are credited and that the original publication in this journal is cited, in accordance with accepted academic practice. No use, distribution or reproduction is permitted which does not comply with these terms. 